Marquette University

e-Publications@Marquette

\title{
Application of a Multi-Zone Model for the Prediction of Species Concentrations in Rapid Compression Machine Experiments
}

David Wilson

Marquette University

\section{Recommended Citation}

Wilson, David, "Application of a Multi-Zone Model for the Prediction of Species Concentrations in Rapid Compression Machine Experiments" (2016). Master's Theses (2009 -). Paper 348.

http://epublications.marquette.edu/theses_open/348 


\section{APPLICATION OF A MULTI-ZONE MODEL FOR THE PREDICTION OF SPECIES CONCENTRATIONS IN RAPID COMPRESSION MACHINE SIMULATIONS}

by

David Wilson, B.S.M.E

A Thesis submitted to the Faculty of the Graduate School, Marquette University, in Partial Fulfillment of the Requirements for the Degree of Master of Science

Milwaukee, Wisconsin

May 2016 


\title{
ABSTRACT \\ APPLICATION OF A MULTI-ZONE MODEL FOR THE PREDICTION OF SPECIES CONCENTRATIONS IN RAPID COMPRESSION MACHINE SIMULATIONS
}

\author{
David Wilson, B.S.M.E
}

Marquette University, 2016

Accurate chemical kinetic models, which predict species evolution and heat release rates in chemically reactive systems, are essential for further advancements in fuel and combustion technology. An experimental facility that is widely used for evaluating the accuracy of kinetic models is a rapid compression machine (RCM), which creates a well-defined reaction environment by compressing a reactive mixture inside a chamber. Generally, RCM experiments are conducted in order to obtain ignition delay data. However, chemical speciation data provides greater insight into reaction pathways, and is therefore a more rigorous benchmark for validating kinetic models.

In order for a chemical kinetic model to be evaluated using RCM data, the kinetic model must be coupled with a thermodynamic model that can predict the temporally varying conditions that evolve during an RCM experiment. The most common approach is to utilize a thermally and compositionally homogeneous 0 -dimensional reactor model (HRM), which predicts conditions inside the hot core region of the main combustion chamber of an RCM, where a significant portion of the chemical reaction in an RCM takes place. This approach requires an effective volume profile, which is derived from the pressure profile of either a non-reactive experiment with similar transport properties as the condition of interest, or a separate multi-zone model (MZM), via the relationship between pressure and volume for an isentropic process. While HRMs have been shown to yield adequate ignition delay predictions, they cannot be used to predict average speciation data, since the conditions in the core region vary considerably from the average conditions of the total reaction chamber.

This work introduces a modified MZM, which simulates chemical reaction throughout the entire temperature-varying main combustion chamber of an RCM, in addition to boundary work, conduction, and crevice flows as the traditional MZM approach. Simulating chemistry in the MZM allows for average speciation predictions, and eliminates the need for an HRM. The new approach is shown to yield similar average speciation data as CFD simulations (within 15\% difference) for the combustion of primary reference fuels at various conditions. 


\title{
ACKNOWLEDGEMENTS
}

\author{
David Wilson, B.S.M.E
}

I would first like to express gratitude to my advisor, Dr. Casey Allen, for all the guidance you have provided throughout these two years. I have genuinely enjoyed this work (so much so that I decided to forgo making an engineer's salary for the next few years so that I can continue doing combustion research at Marquette), and much of that is credited to you. Your willingness to share knowledge and provide guidance through the various obstacles that I faced, even at times when you were busy, gave me the confidence I needed to complete my project. I'm looking forward to our future projects, and I hope we make can a significant impact in field of combustion.

I would also like to thank the graduate students who have come before me, specifically John Neuman and Benyamin Davaji, who have become dear friends to me since I first arrived at Marquette. Both of you made me feel right at home from the time I first set foot in the graduate study room. The laughs we shared helped me maintain balance throughout my work, and made the office a genuinely fun place to be. You guys also taught me how to be a graduate student, and how to balance research and class work. I am grateful for our friendship.

Thank you to the current members of our research group: Jack Rehn and Jenna Ezzell, for your research efforts and your friendship, both of which have helped my own research efforts. I look forward to continue working with you two and building our friendship.

Thanks to my thesis committee: Dr. John Borg and Dr. Simcha Singer, for agreeing to be on the committee and making efforts to provide feedback for my research.

Last, but not least, I surely owe the biggest thanks to my family, who have supported me and believed in me not only throughout my time at Marquette, but throughout my entire life. If it wasn't for your love and guidance, I surely would not be where I am today. 


\section{TABLE OF CONTENTS}

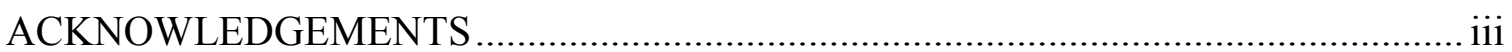

LIST OF TABLES ............................................................................................. vii

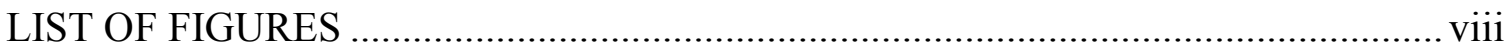

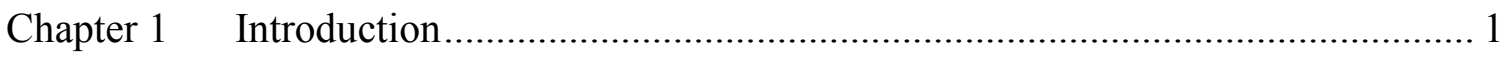

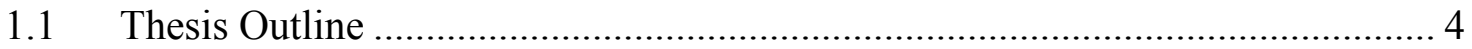

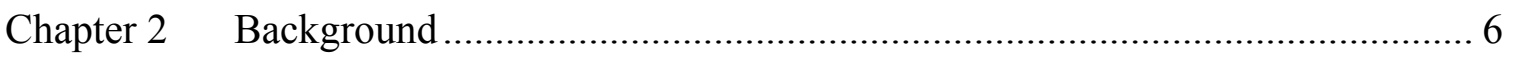

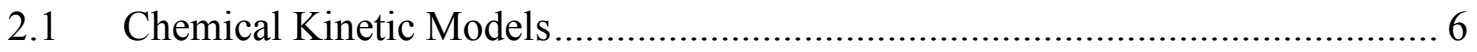

2.1.1 Mathematical Model .......................................................................... 6

2.1.2 Applications .................................................................................. 7

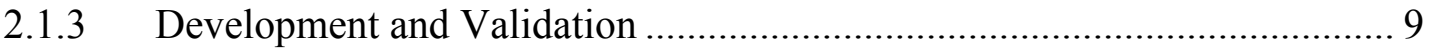

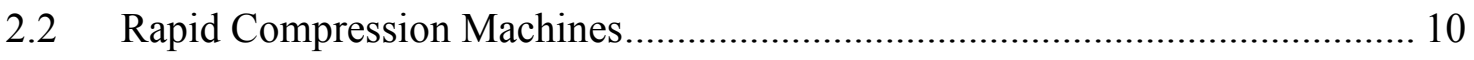

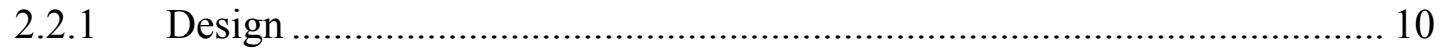

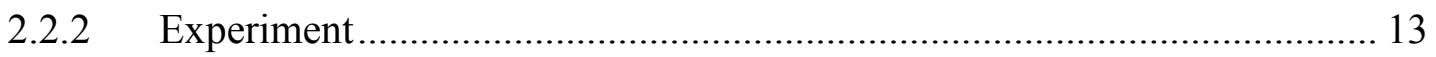

2.3 Rapid Compression Machine Modeling..................................................... 15

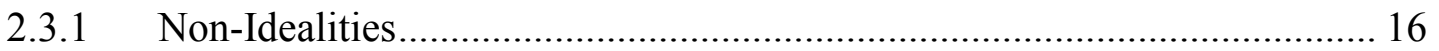

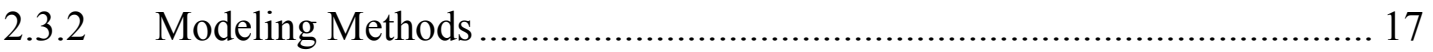

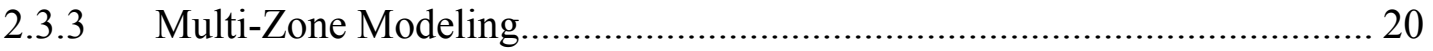

2.3.3.1 Original Multi-zone Model.......................................................... 20

2.3.3.2 Multi-Stage Ignition Modeling ................................................. 22

2.3.3.3 New MZM Model.................................................................. 24

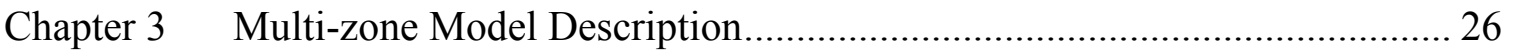

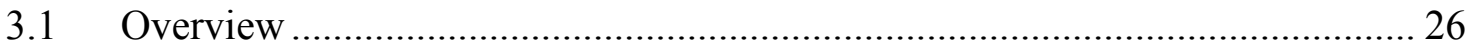




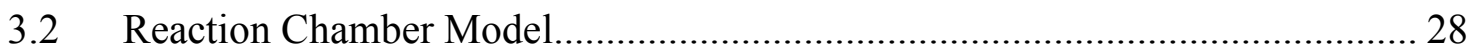

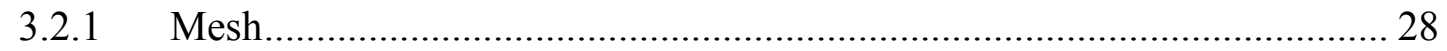

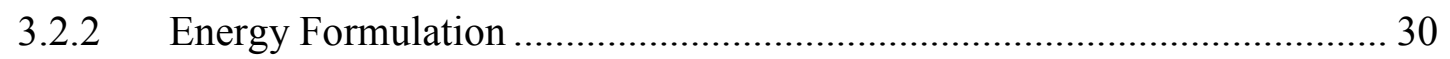

3.2.2.1 Energy Equation for a Single Zone ………........................................ 30

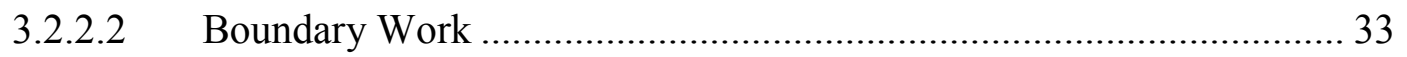

3.2.2.3 Conductive Heat Transfer.................................................................. 34

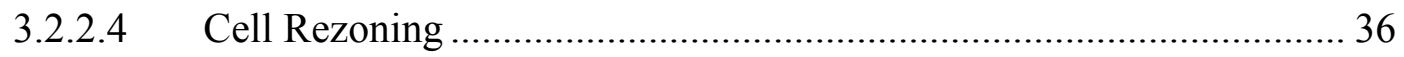

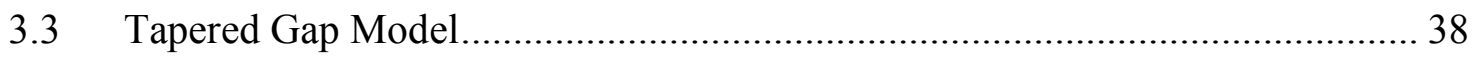

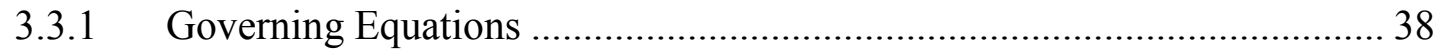

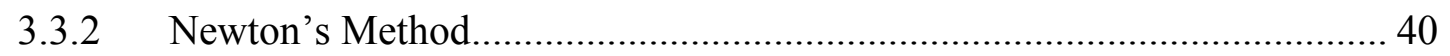

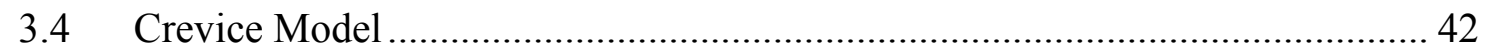

3.4.1 Governing Equations ……………………...................................... 42

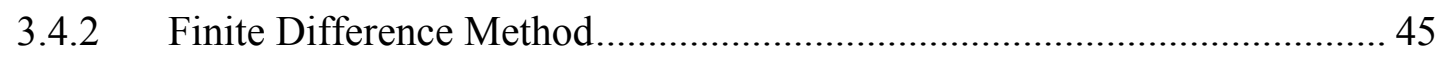

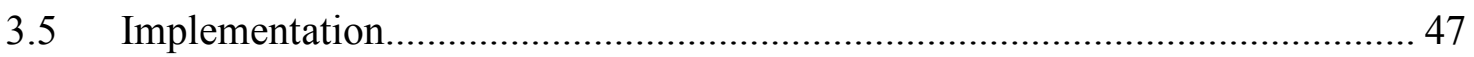

Chapter $4 \quad$ Multi-zone Model Validation ................................................................. 51

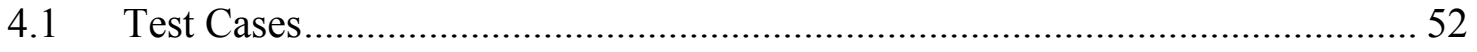

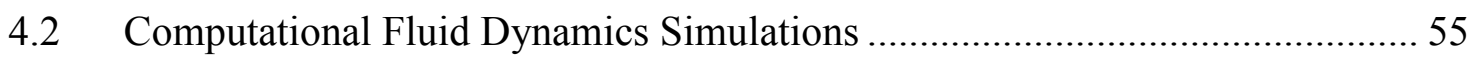

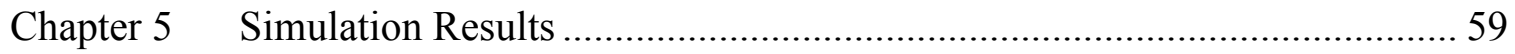

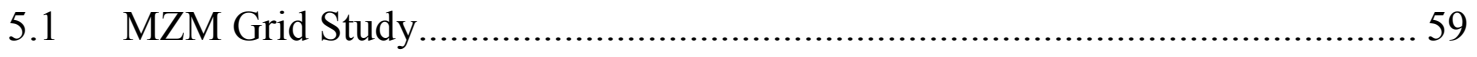

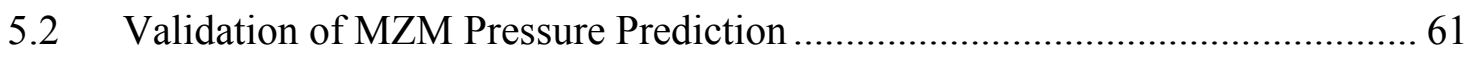

5.3 Validation of MZM Maximum Temperature Prediction....................................... 65

5.4 Temperature Distribution Comparison between MZM and CFD ....................... 69

5.5 Validation of MZM Chemical Species Prediction ........................................... 72 


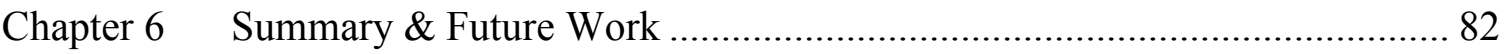

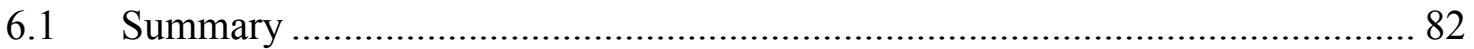

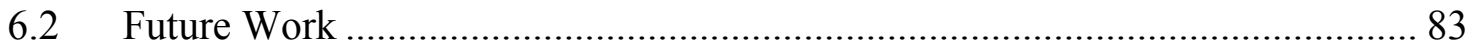

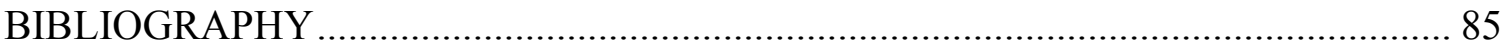




\section{LIST OF TABLES}

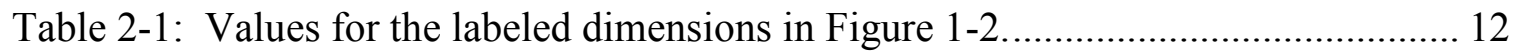

Table 4-1: An overview of the conditions for each test case for which MZM and CFD simulations are performed.............................................................................. 53

Table 5-1: Ignition delay times for each case for MZM, HRM, and CFD simulations... 73 


\section{LIST OF FIGURES}

Figure 2-1: RCM Schematic: a) Top-down view b) Side view [Neuman, 2015]........... 11

Figure 2-2: 2-D sector schematic of the piston and reaction chamber in the RCCEM at

Marquette University. .......................................................................... 12

Figure 2-3: Illustration of the boundary layer shearing effect for a flat piston versus a crevice piston. [Sung \& Curran, 2014] .......................................................... 17

Figure 3-1: A 1-dimensional grid representation of the reaction chamber of an MZM, sliced along the axis of symmetry. .............................................................. 29

Figure 3-2: A 1-dimensional illustration of an MZM grid with 6 reaction chamber zones, sliced along the axis of symmetry. 33

Figure 3-3: Illustration of conductive heat transfer for an MZM zone. 35

Figure 3-4: Example illustration of the cell rezoning technique for the third innermost zone in an MZM reaction chamber mesh.

Figure 4-1: 30-degree sector computational domain utilized for CFD simulations [Neuman, 2015]

Figure 5-1: MZM maximum temperature versus time for case 6 for varying numbers of zones.

Figure 5-2: MZM pressure versus time for case 6 for varying numbers of zones.

Figure 5-3: Predicted main reaction chamber pressures for MZM and CFD RCM simulations with a stoichiometric mixture of $n$-heptane and air at compression ratios of 9,10 and 11

Figure 5-4: Predicted main reaction chamber pressures for MZM and CFD RCM simulations with a stoichiometric mixture of $i s o$-octane and air at compression ratios of 11,12 , and 13 .

Figure 5-5: Predicted main reaction chamber pressures for MZM and CFD RCM simulations with a stoichiometric mixture of fuel and air at compression ratios of 9,10 , and 11 . The fuel is a mixture of $50 \%$ by volume $i s o$-octane and $50 \%$ by volume $n$-heptane.

Figure 5-6: Maximum main reaction chamber temperature for RCM simulations with a stoichiometric mixture of $n$-heptane and air at compression ratios of 9,10 and 11 . 
Figure 5-7: Maximum main reaction chamber temperature for RCM simulations with a stoichiometric mixture of $i s o$-octane and air at compression ratios of 11,12 , and 13.

Figure 5-8: Maximum main reaction chamber temperature for RCM simulations with a stoichiometric mixture of fuel and air at compression ratios of 9,10 , and 11 . The fuel is a mixture of $50 \%$ by volume iso-octane, and $50 \%$ by volume $n$-heptane. 68

Figure 5-9: Fraction of main reaction chamber volume occupied by single temperatures for the MZM, and temperature ranges for CFD, for the iso-octane, compression ratio of 11 case.

Figure 5-10: MZM, HRM, and CFD predictions of mole fractions of $n$-heptane $\left(\mathrm{C}_{7} \mathrm{H}_{16}\right)$, oxygen $\left(\mathrm{O}_{2}\right)$, formaldehyde $\left(\mathrm{CH}_{2} \mathrm{O}\right)$, and hydroxyl $(\mathrm{OH})$ radical for stoichiometric $n$-heptane/air cases.

Figure 5-11: MZM, HRM, and CFD predictions of mole fractions of iso-octane $\left(\mathrm{C}_{8} \mathrm{H}_{18}\right)$, oxygen $\left(\mathrm{O}_{2}\right)$, formaldehyde $\left(\mathrm{CH}_{2} \mathrm{O}\right)$, and hydroxyl $(\mathrm{OH})$ radical for stoichiometric iso-octane/air cases. 78

Figure 5-12: MZM, HRM, and CFD predictions of mole fractions of $n$-heptane $\left(\mathrm{C}_{7} \mathrm{H}_{16}\right)$, iso-octane $\left(\mathrm{C}_{8} \mathrm{H}_{18}\right)$, oxygen $\left(\mathrm{O}_{2}\right)$, formaldehyde $\left(\mathrm{CH}_{2} \mathrm{O}\right)$, and hydroxyl $(\mathrm{OH})$ radical for cases with a stoichiometric mixture of a fuel blend and air. 


\section{Chapter 1 Introduction}

Chemical kinetic models, which predict species evolution and heat release rates in chemically reactive systems, are a key component of engine simulations and are therefore critical for further advancements in engine design. In order for the predictive accuracy of a chemical model to be verified, it must produce results similar to those obtained from experiments. One way to generate data for chemical kinetic model validation is to conduct rapid compression machine (RCM) experiments. RCMs produce a well-defined reaction environment by rapidly compressing a reactive gas mixture with a piston inside a piston cylinder. The typical parameter that is measured in RCM experiments is ignition delay time, which is the time that passes between the end of compression and the initiation of combustion of the reactive mixture (indicated by the maximum pressure rise with respect to time). Another parameter which provides a more rigorous benchmark for validating chemical models is speciation data. Intermediate species concentrations provide direct insight into exactly what chemical models are trying to predict: chemical evolution rates and reaction pathways. Speciation data in an RCM experiment is often obtained by expanding the reactive mixture at a specific time in the post-compression period, effectively quenching the mixture and terminating any further chemical evolution.

In order to validate a particular chemical model using RCM data, the chemical model must be coupled with a thermodynamic model that can predict the temporally varying conditions that evolve during an RCM experiment. Computational fluid dynamics (CFD) can be used for this purpose. However, CFD simulations are very computationally expensive, and cannot be used to simulate a wide range of conditions in a reasonable amount of time. More often, a thermally and compositionally homogeneous 
0-dimensional reactor model (HRM) is used to predict the conditions inside the hot core region of the main combustion chamber of an RCM. This approach is very fast and simple, as it employs only a single zone. Here, boundary work is applied to the zone according a specific volume profile. The volume profile is derived from the pressure profile of either a non-reactive experiment with similar transport properties as the condition of interest, or a separate multi-zone model (MZM), via the relationship between pressure and volume for an isentropic process.

An MZM predicts the temporally and spatially varying conditions within the main combustion chamber during an RCM experiment by modelling physical processes that occur, such as boundary work, conductive heat loss, and mass flow to the crevice. MZM simulations are much simpler and computationally efficient than CFD simulations for several reasons. For one, the computational domain in an MZM consists of far fewer cells (20 cells were used for the simulations presented in this thesis). This is because the MZM neglects multi-dimensional effects within the main reaction chamber, allowing the main reaction chamber to be treated as one-dimensional where the conditions vary as a function of distance from the boundaries (piston and cylinder wall). Therefore, the MZM mesh can be formulated with concentric, cylindrical zones. Modelling the main reaction chamber in such a way is satisfactory due to the presence of a crevice machined into the side an RCM piston, which has been shown to effectively swallow boundary layer gas which shears off the cylinder walls, which would otherwise permeate the core of the main reaction chamber. Also, convective heat loss is neglected in the MZM, since the crevice reduces advection within the main reaction chamber. Lastly, since the spatially varying conditions within machined crevice and the gap between the main reaction chamber and 
crevice are unimportant in regard to ignition characteristics within an RCM, since minimal chemical reaction occurs in these regions, they are each formulated as a single zone which reflects the average conditions in these regions.

While HRMs have been shown to yield accurate predictions of ignition delay time and conditions within the core region of the main reaction chamber for experiments with a single stage of ignition, they cannot be used to predict speciation data for an RCM experiment. This is because average speciation data throughout the entire reaction chamber, which is what is measured in quenching experiments, is much different than that in the core. Also, the HRM fails to predict accurate ignition delay data for cases where significant chemical heat release occurs before the main combustion event, as the effects of heat release are not present in the effective volume profiles obtained from a non-reactive simulation or MZM. As a result, a modified MZM has been formulated for predicting average speciation data in an RCM experiment. The modified MZM is suitable for this purpose because it simulates chemical evolution and heat release in each main reaction chamber cell, allowing spatially varying reaction rates to be calculated and eliminating the need for an HRM. This also allows for the effects of chemical heat release that occur during a simulation to be directly accounted for. The modified approach is shown to yield great improvement in average speciation data for an RCM experiment. This thesis mainly describes the modified MZM model, as well as presents simulation results yielded from the model. An outline of the thesis is presented below in the following sub-section. 


\subsection{Thesis Outline}

The thesis begins with a background section which first describes how chemical kinetic models calculate chemical evolution rates, and the importance of chemical kinetic models in engine and combustion research. A brief description of how a chemical model may be designed is then provided, followed by an outline of different experimental methods that are used to validate chemical models. Next, RCM design and experimentation are discussed, and descriptions for how ignition delay and speciation data are obtained are provided. The chapter concludes with a discussion of modelling approaches that are used to simulate and predict the thermodynamic conditions inside an RCM, including the HRM and MZM. A literature review on how these two modelling approaches have been applied and the different variations in which they exist is provided.

Chapter 3 introduces the modified MZM, which has been reformulated to allow for predictions of average species concentrations in an RCM experiment. The chapter begins with a general overview of the modified MZM and the description of the assumptions necessary for formulate the model. This is followed by a description of the main reaction chamber mesh generation technique. Next, the energy equation as applied to a single MZM zone is formulated, and the different energy interactions that can occur for a zone are described. The cell rezoning method for ensuring that pressure is equilibrated in each main reaction chamber zone is then provided. Next, the tapered gap model is described, including the application of Newton's method to calculate the mass flow rate through the gap. The chapter concludes with a description of the crevice submodel, and the finite difference formulas that are used to determine the mass, velocity, and internal energy of the crevice zone at a subsequent time. 
Chapter 4 describes the process for evaluating the predictive accuracy of the MZM, which involves comparing temperature, pressure and speciation data yielded by the MZM with data from CFD for various conditions. CFD data is used as a benchmark for evaluating the MZM, since CFD is the most rigorous numerical method available for simulating RCM experiments. The test conditions at which the simulations were conducted, as well as reasoning for choosing each particular condition is given. A description of the chemical kinetic model used for the simulations is also provided.

Chapter 5 provides data from simulations of the combustion of iso-octane and $n$ heptane for the modified MZM and CFD. First, the results for a study used to determine mesh independence for the MZM is provided. The temperature and pressure profiles yielded by the MZM and CFD for each of the test cases given in Chapter 4 are then provided and discussed. This is followed by a comparison of the temperature distribution between MZM and CFD for the case with the greatest discrepancy in the results between the two simulations. Chemical speciation data for each simulation is then presented and compared.

Chapter 6 provides a summary of the thesis, as well as provides directives for future work. These include ideas for improving the model and the suggestion to perform RCM experiments for further evaluating the accuracy of the model. 


\section{Chapter 2 Background}

\subsection{Chemical Kinetic Models}

\subsubsection{Mathematical Model}

Chemical kinetic models predict the temporal progression of chemical reaction in a system. A kinetic model consists of various chemical species and elementary reactions. Each reaction in the model is assigned a particular rate constant at a given time in a kinetic simulation. The rate constant is generally assumed to be a function of temperature only, which is a valid assumption for moderate temperature ranges, and is calculated using the modified Arrhenius rate equation [Turns, 2012]:

$$
k=A T^{b} \exp \left(-\frac{E_{a}}{R T}\right)
$$

The rate constant is represented by $k, T$ is the temperature, $R$ is the universal gas constant, and $A, b$, and $E_{a}$ are empirical parameters. With $k$ known, the rate of each reaction can be calculated accordingly [Turns, 2012]:

$$
q_{r}=k \prod_{j=1}^{N}\left[X_{j}\right]^{v_{j r}}
$$

Subscripts $r$ and $j$ are indices representing a given reaction and species in the model,

respectively. Variable $N$ represents total number of species in the model, $\left[X_{j}\right]$ represents molar concentration of a particular species, and $v_{j i}$ represents the stoichiometric coefficient of a given species in a given reaction. The production rate of each species is calculated as follows [Turns, 2012]:

$$
\dot{\omega}_{j}=\sum_{r=1}^{L} v_{j r} q_{r}
$$


The total number of reactions in the mechanism is represented by $L$. Equation 2-3 is then integrated to calculate the molar fraction or molar count of each species at a subsequent time.

\subsubsection{Applications}

The applicability of chemical kinetic models for predicting chemical evolution in physical systems extends a wide range of science and engineering fields. A few examples of these fields include the combustion of conventional and alternative fuels [Simmie, 2003], astrochemistry [Costes \& Naulin, 2013], and drug design [Tiwary, Limongelli, Salvalaglio, \& Parrinello, 2015]. Depleting fossil fuels as well as the negative environmental impacts associated with the combustion of petroleum based fuels have placed increased importance on improving chemical kinetic models for engine and alternative fuel design. Chemical kinetic models aid in the development of engines by predicting important ignition characteristics (such as ignition delay, energy release rate, and chemical species formation). For spark-ignition engines, knock occurrence, which limits the compression ratio and therefore the efficiency at which a spark-ignition can operate, has been investigated with the use of numerical modeling and chemical models [Liang, L., Reitz, R., Iyer, C., and Yi, 2007; Linse, Kleemann, \& Hasse, 2014]. The relationship of soot and nitrogen oxide $\left(\mathrm{NO}_{\mathrm{x}}\right)$ formation with various conditions within a diesel engine such as temperature, pressure and flame lift-off length, as well as design parameters such as spray nozzle geometry has also been explored using chemical kinetics [Kong, Sun, \& Rietz, 2007; Som, Ramirez, Longman, \& Aggarwal, 2011]. There exist numerous other studies in the literature that utilized chemical kinetic models to predict engine performance. 
Chemical kinetic models are especially pertinent to the further development of novel engine concepts, such as the homogeneous charge compression ignition (HCCI) engine. An HCCI engine can be superficially described as a combination of a sparkignition (SI) and a diesel or compression ignition (CI) engine, utilizing the best characteristics of each. As in an SI engine, fuel is injected into an HCCI engine during the intake stroke. This allows sufficient time for the fuel to thoroughly mix with the air in the cylinder, hence the "HC" (homogeneous charge) in HCCI. As in a diesel engine, combustion is initiated by the temperature rise due to compression.

The HCCI engine is advantageous in terms of efficiency and emissions. First of all, HCCI engines are not plagued by the throttling losses that are characteristic of SI engines. SI engines require that the air-to-fuel ratio be approximately stoichiometric, regardless of load, in order to utilize a three-way catalytic converter to limit $\mathrm{HC}, \mathrm{CO}$ and NO emissions [Heywood B. John, 1988]. This is achieved by restricting airflow to the cylinder at the expense of introducing throttling losses. Also, the compression ratio at which HCCI engines can operate is not limited by knocking phenomenon, unlike SI engines. Lastly, harmful particulate matter and $\mathrm{NO}_{\mathrm{x}}$ emissions from an $\mathrm{HCCI}$ are much lower than that of diesel engines, due to the homogeneous mixing of air and fuel and lower combustion temperatures [Epping, Aceves, Bechtold, \& Dec, 2002]. One of the main setbacks preventing HCCI engines from transitioning from laboratories to industry is that the performance of HCCI engines is very sensitive to combustion timing and heat release rate, which are governed by chemical kinetics and are difficult to predict and control. Therefore, models that can accurate predict chemical kinetics are essential for the advancement of HCCI engines [Saxena \& Bedoya, 2013]. 


\subsubsection{Development and Validation}

In a very basic sense, the design of chemical kinetic models involves choosing which reactions to include in the model and assigning specific rate constants $(A, b$, and $E_{a}$ from Equation 2-1) to each reaction. The chemical model design process is complicated, and relies heavily on the intuition of kinetic experts, and general rules of thumb. Also, many chemical models are made for a specific purpose, such as the combustion of a certain fuel in a certain temperature and pressure range. Most models involving hydrocarbon combustion are designed in a hierarchical fashion, with chemistry involving hydrogen, oxygen and carbon monoxide at the base [Simmie, 2003].

Chemical kinetic models must be validated with experimental data over a wide range of conditions in order to ensure their accuracy and ability to predict chemical evolution in a real system. Flow reactor [Dayma et al., 2011], shock tube [Yasunaga et al., 2012], laminar flame [Ranzi et al., 2012], and rapid compression machine [Sung \& Curran, 2014] experiments are all typical for evaluating chemical models. Each of the aforementioned experimental method aims at creating a homogeneous reaction environment and reducing convective and diffusive effects, in order to eliminate uncertainty and isolate the effects of chemical kinetics. A brief overview of flow reactor, shock tube and laminar flame experiments is provided in the next few paragraphs. A more in depth discussion rapid compression machine experiments and modeling are provided in the next subsection.

In a flow reactor experiment, a well-mixed air-fuel mixture flows into a reaction chamber, where the mixture is heated to a known temperature in order to induce combustion. The chemical composition of the mixture can then be determined through 
chromatography and spectroscopy methods applied to samples. Typical flow reactor experiments occur at intermediate pressures, from atmospheric to about 15 bar [Mueller, Yetter, \& Dryer, 1999].

A shock tube is a long cylinder consisting of a pressurized driver section, and a driven section, where the mixture of interest is located. The sections are separated by a diaphragm. In a shock tube experiment, the diaphragm is broken, creating an initial as well as incident shock wave through the driven section, almost instantaneously compressing the mixture, which initiates combustion. Ignition delay, pressure and species concentrations are often measured in shock tube experiments.

In laminar flame experiments, fuel at the base of a burner reacts with surrounding air, ideally creating a one-dimensional "flat" flame inside a chamber from which samples are extracted and expanded into a vacuum. These samples are then analyzed using a mass spectrometry method in order to determine the chemical composition. Most laminar flame experiments are conducted at pressures near 1 bar. Difficulties achieving flame stability occur at higher pressures [Egolfopoulos et al., 2014].

\subsection{Rapid Compression Machines}

\subsubsection{Design}

Rapid compression machines (RCMs) generate a well-defined reaction environment by rapidly compressing a reactive gas mixture inside a reaction chamber using a piston, and then maintaining a constant volume during post compression. The low-temperature, high-pressure conditions inside an RCM closely reflect the conditions 
inside engines and gas turbines, making RCM data valuable for validating chemical models for engine simulations. A typical RCM design consists of a driving mechanism, braking mechanism, and a reaction chamber. One variation of an RCM is a rapid compression controlled-expansion machine (RCCEM), which allows retraction of the piston after compression, effectively expanding the reactive mixture. This capability provides a way to explore the effects of heat loss during an experiment, since both heat loss and expansion result in lower temperatures in the reaction chamber. The machine at Marquette University, shown in Figure 1, is an RCCEM. In the RCCEM, a pneumatic actuator drives a cam using compressed air. As the cam moves forward, it drives piston, which compresses the reactive mixture in the combustion chamber. The piston profile is determined by the shape of the cam. This is advantageous, because it allows different piston profiles, such as ones that include expansion, to be created by simply switching cams [Neuman, 2015].

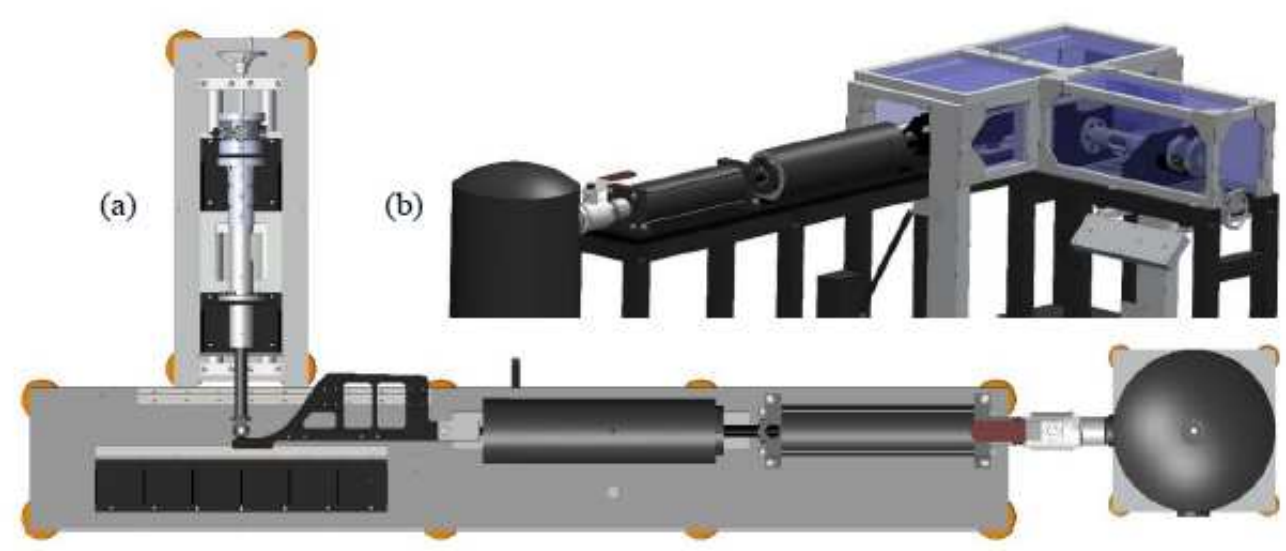

Figure 2-1: RCM Schematic: a) Top-down view b) Side view [Neuman, 2015] 
The simulations presented in this thesis utilize the geometry of the reaction chamber in the RCCEM at Marquette. Therefore, the reaction chamber geometry is presented. Figure 1-2 provides an illustration of reaction chamber and piston, with corresponding dimensions given in Table 1-1. It should be noted that although the machine for which the simulations are based is an RCCEM, the simulation results presented in this thesis are for experiments with compression only.

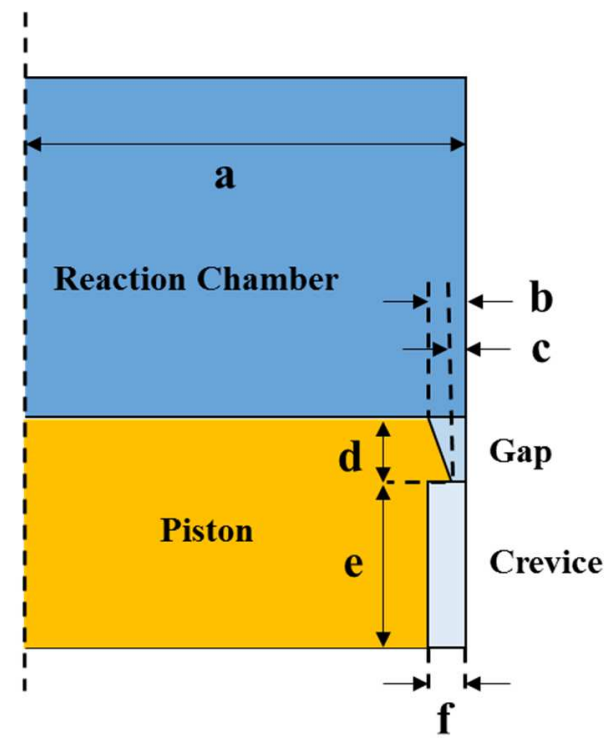

Figure 2-2: 2-D sector schematic of the piston and reaction chamber in the RCCEM at Marquette University. Note, this image is not to scale.

\begin{tabular}{|c|c|}
\hline Dimension & Value (in) \\
\hline a & 1.0 \\
b & 0.022 \\
c & 0.009 \\
d & 0.157 \\
e & 0.786 \\
f & 0.70 \\
\hline
\end{tabular}

Table 2-1: Values for the labeled dimensions in Figure 1-2. 


\subsubsection{Experiment}

In RCM experiments, the most common parameter that is measured is pressure. Pressure measurement is accomplished using a pressure transducer that is attached to the reaction chamber. The pressure data is used to determine ignition delay time, which is defined as the elapsed time between the end of compression and the time at which the derivative of pressure with respect to time $\left(\frac{d P}{d t}\right)$ is maximum [Sung \& Curran, 2014]. Ignition delay data are often obtained for a wide range of compressed temperatures $\left(T_{c}\right)$, compressed pressures $\left(P_{c}\right)$ and air-to-fuel ratios, in order to validate a mechanism across a wide range of conditions. Since reaction kinetics are strongly dependent on temperature, it is desirable to have temperature data from RCM experiments. However, direct temperature measurements are very challenging, due to the rapidly changing conditions inside an RCM, and time scale limitations for thermocouples.

Temperature in an RCM experiment can be calculated using the "adiabatic core" hypothesis, which assumes that the effects of heat loss only pertain to the boundary layer region along the piston and cylinder walls. The core region is assumed to be isothermal and undergo only adiabatic and isentropic processes [Sung \& Curran, 2014]. These assumptions allow the compressed temperature to be estimated using the relationship between temperature and pressure for an isentropic process:

$$
\int_{T_{0}}^{T_{C}} \frac{\gamma}{\gamma-1} \frac{d T}{T}=\ln \left(\frac{P_{C}}{P_{0}}\right)
$$

Variables $P_{0}$ and $T_{0}$ represent the initial pressure and temperature in an RCM experiment, and $\gamma$ represents the temperature dependent specific heat ratio, which varies with time. 
RCM experiments are primarily conducted in order to obtain ignition delay data, which is often presented versus compressed temperatures. While ignition delay measurements are an important benchmark for confirming the ability of a mechanism to predict global reaction rates, such as the rate at which a fuel is consumed during combustion, they do not by themselves provide direct insight into reaction pathways. For example, the RAMEC chemical mechanism [Petersen, Davidson, \& Hanson, 1999], and LEEDS mechanism [Hughes, Turanyi, Clague, \& Pilling, 2001], both of which were designed for the combustion of methane, predict similar ignition delays under identical conditions, but use very different rate constants for important elementary reactions. This suggests that oxidation of simple fuels, such as methane, are not truly understood on the elementary level [Simmie, 2003].

Unlike ignition delay, chemical speciation data is a direct reflection of exactly what mechanisms are trying to predict: reaction rates and species evolution. There exists studies that have obtained speciation data from RCM "quenching" experiments, where the contents inside an RCM are rapidly cooled in order to terminate further chemical reaction [Vanhove et al., 2015; Wagnon, Barraza-Botet, \& Wooldridge, 2015]. Quenching involves expanding the reactive gas mixture at a specific time during the ignition delay period, effectively cooling the gas and terminating further chemical reaction. This is often accomplished by having a vacuumed chamber next to the main reaction chamber, separated by a diaphragm, and then rupturing the diaphragm at a specific time. Gas chromatography and mass spectroscopy is then used to analyze the chemical contents of the sample. 
Once data from an RCM experiment is collected, it can be used to evaluate the predictive accuracy of a chemical kinetic model. This evaluation process involves simulating an RCM experiment with a thermodynamic model coupled with the chemical kinetic model of interest. The results yielded from the simulations are then compared with the experimental results. Typically, the results are compared in terms of temperature, ignition delay, and species concentrations. A chemical model is considered validated if it reproduces the results seen in experiments over a range of conditions. An RCM simulation requires a thermodynamic model which is capable of capturing the physical processes that occur inside the reaction chamber, such as mass transfer between the reaction chamber and the cooler crevice and heat loss to the boundaries. An overview of the various approaches for modeling RCM experiments is given in the following subsection.

\subsection{Rapid Compression Machine Modeling}

Each experimental method for studying chemical kinetics aims to create a homogeneous reaction environment, void of convective effects and heat loss, so that reaction rates determined from an experiment can be easily characterized with respect to temperature and chemical composition. However, non-ideal effects are present in every experimental method, including RCM experimentation. These effects must be accounted for in the modeling approaches, since they significantly affect temperature distribution within a reactive system, and therefore reaction rates. Non-idealities within an RCM experiment are discussed below, followed by a description of various modeling approaches for simulating RCM experiments. 


\subsubsection{Non-Idealities}

Early RCM designs incorporated a flat piston head, which results in a roll-up vortex in the reaction chamber that is caused by motion of the piston during compression. This vortex shears along the reaction chamber walls toward the piston, and inwardly along the piston head. As the vortex shears along the piston head, it begins to penetrate into the core region of the reaction chamber, introducing cold boundary gas into the core. This is problematic, as it eradicates the validity of the adiabatic core hypothesis, and therefore makes determining temperature and overall state in the reaction chamber difficult [Clarkson, Griffiths, MacNamara, \& Whitaker, 2001].

Current RCM designs employ a creviced-piston in order to combat the effects of the vortex. The crevice swallows the boundary layer gas that shears off the reaction chamber walls, preventing further shearing along the piston head and penetration into the core [Lee \& Hochgreb, 1998]. This design has been shown to greatly improve homogeneity and reduces convective effects within the reaction chamber [Mittal \& Sung, 2006]. This is advantageous for reducing the complexity involved in modeling. However, it also introduces another phenomenon that has to be accounted for: mass flow to the crevice. It is especially important that mass flow to the crevice is modeled for cases with multi-stage ignition, where ignition can cause additional mass to flow from the reaction chamber to the crevice [Mittal, Raju, \& Bhari, 2011]. Figure 1-3 illustrates the roll-up vortex and its suppression using a creviced piston. 


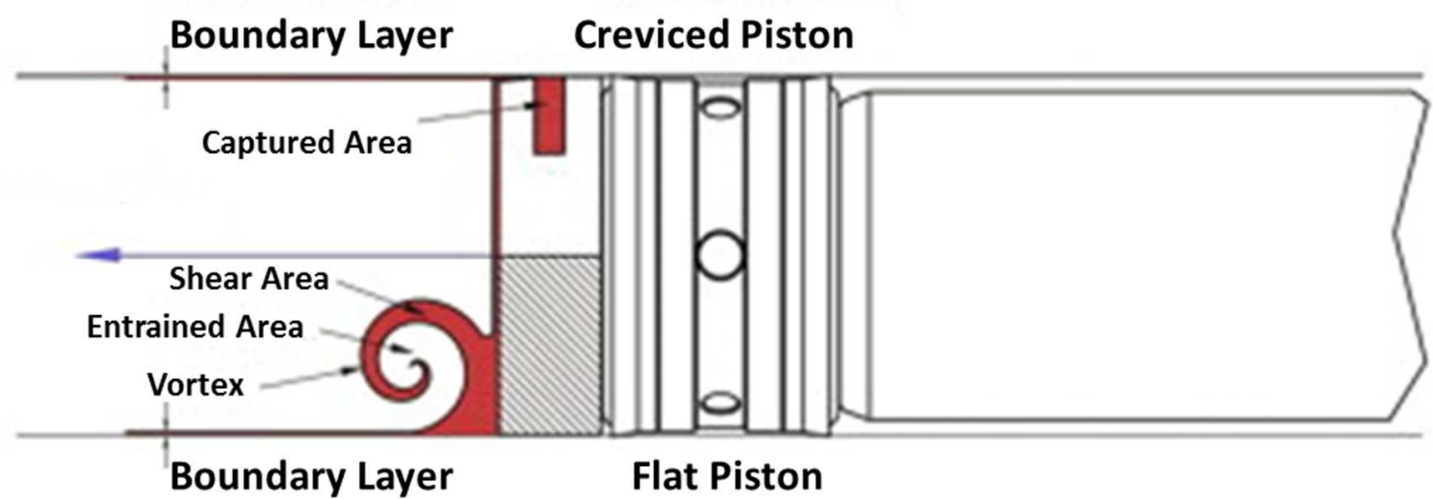

Figure 2-3: Illustration of the boundary layer shearing effect for a flat piston versus a crevice piston. [Sung \& Curran, 2014]

Another inevitable non-ideality that reduces core temperature and produces temperature inhomogeneity in RCM experiments is heat loss to the boundaries. This results from reaction chamber walls being a lower temperature than the reacting mixture. There are a variety of modeling methods for RCM experiments that attempt to account for non-idealities explained above. These modeling methods are explained below.

\subsubsection{Modeling Methods}

The most rigorous modeling method for RCM experiments is computational fluid dynamics (CFD), which directly accounts for heat loss and gas dynamic effects. There exist studies that investigate the gas dynamics within the reaction chamber of an RCM experiment using CFD [Mittal, Raju, \& Sung, 2012; Würmel \& Simmie, 2005]. However, CFD simulations are incredibly computationally expensive, making this method intractable for simulating the wide range of conditions necessary for validating a chemical kinetic model. The chemical kinetic equations need to be solved for each of the numerous cells in a CFD computational domain. This intractability problem magnifies 
for large kinetic mechanisms, some of which contain well over 1000 different species and reactions [Herbinet, Pitz, \& Westbrook, 2010].

Another modelling approach is to utilize a homogeneous reactor model (HRM), which is a single zone with uniform conditions that is meant to represent the core of an $\mathrm{RCM}$ reaction chamber. Modelling with an HRM is attractive due its simplicity, which results in significant reduction in computational costs. However, in order for an HRM to accurately simulate conditions in the core, it must employ a method to account for heat loss.

One approach for modeling the heat loss during post-compression for an HRM is to add an effective heat loss term to the energy equation of the HRM [Ribaucour et al., 2000; Westbrook et al., 2002]:

$$
\left.\frac{d U_{H R M}}{d t}\right]_{\text {heat loss }}=h_{e f f} A_{H R M}\left(T_{\text {wall }}-T_{H R M}\right)
$$

The variable $U_{H R M}$ represents the internal energy of the HRM, $h_{\text {eff }}$ is an effective heat transfer coefficient, and $A_{H R M}$ is the surface area of the HRM. In this approach, the volume of the HRM is assumed to remain constant during post-compression. The effective heat transfer coefficient is estimated using pressure traces from non-reactive experiments, since the pressure traces have to be void of chemical ignition effects to determine the effect of heat loss on pressure. Temperature is calculated from pressure using the ideal gas law for constant volume: $T(t)=T_{c}\left[P(t) / P_{c}\right]$. Compressed temperature is calculated from Equation 2-4. While this method is simple, it significantly over-predicts heat loss from the core region compared to experiments and CFD simulations [Sung \& Curran, 2014]. 
Another method for accounting for heat loss for the HRM during postcompression, which yields significantly better agreement with experiment and CFD, is to maintain the adiabatic core hypothesis during post-compression in addition to the compression period. This assumption results in an isentropic expansion of the core gas in response to heat loss from boundary layer to the walls [Tanaka, Ayala, \& Keck, 2003]. No heat is lost from the core region, rather the core just expands so that the pressure of the core and boundary layer are equilibrated. As a result, an effective volume profile can be calculated from a non-reactive experimental pressure trace:

$$
V_{H R M}(t)=V_{H R M, 0}\left[\frac{P_{H R M, 0}}{P(t)}\right]^{\frac{1}{\gamma}}
$$

Here, the pressure values are determined from a non-reactive experiment, while the initial volume of the HRM can be arbitrarily chosen. The volume profile obtained from Equation 2-6 determines the boundary work $\left(\frac{d V}{d t}\right)$ in the energy equation of the HRM.

While the isentropic volumetric expansion method is an accurate way to account heat loss during post-compression of an RCM experiment, it requires a method for determining an appropriate pressure profile. This can be accomplished by conducting non-reactive experiments with the same initial conditions and specific heat ratio as the reactive experiment of interest. Non-reactive experiments are often achieved by replacing oxygen with nitrogen [Mittal \& Sung, 2007]. Another method for obtaining a pressure profile is to use a modeling approach. 


\subsubsection{Multi-Zone Modeling}

\subsubsection{Original Multi-zone Model}

One example of a modeling approach to account for heat loss is the physics-based multi-zone model (MZM) proposed by Goldsborough et al. [Goldsborough, Banyon, \& Mittal, 2012]. With this approach, the pressure profile for an RCM experiment is calculated from the MZM. The pressure profile is then used to determine the volume profile for an HRM simulation. In the MZM, physical process such as boundary work from compression, conduction heat transfer throughout the reaction chamber and to the piston and walls, and mass transfer between the reaction chamber and crevice are directly modeled. Specifics regarding how these physical processes are modeled in the MZM proposed by Goldsborough et al. are not reviewed here, since the MZM proposed in this thesis is very similar, and is explained in detail in Chapter 3. The MZM was shown to be effective for predicting temperature and pressure evolution for non-reacting simulations by yielding similar results as CFD simulations. Accurate ignition delay predictions were also obtained for a few cases.

In the approach by Goldsborough et al., the pressure derived from the MZM is fed into the differential form of the isentropic relationship between volume and pressure, in order to obtain a volume profile for the HRM:

$$
\frac{d V}{d t}=\frac{V^{\gamma+1}}{\gamma}\left[\frac{\ln (V)}{V^{\gamma+1}} \frac{d \gamma}{d t}+\frac{1}{C} \frac{d P}{d t}\right]
$$

Equation 2-7 is similar to Equation 2-6. They are both the isentropic relationship of volume and pressure. The main differences are that Equation 2-7 is in the differential form, and takes into account the effect of varying specific heat ratio $(\gamma)$ throughout time. 
Constant $C$ is set using the initial conditions of the HRM and the standard form of the isentropic relationship with pressure: $P_{0} V_{0}{ }^{\gamma_{0}}=C$. Again, the initial volume can be arbitrarily chosen. The specific heat in Equation (2-7) is representative of conditions in the HRM, while pressure derivative comes from the MZM. Pressure in the HRM should be greater than the pressure in the MZM, due to chemical heat release.

Pressure in the reaction chamber is found in the MZM using the energy equation as follows:

$$
\dot{Q}-P \frac{d V}{d t}+h^{\prime} \frac{d N}{d t}=u^{\prime} \frac{d N}{d t}+N c_{v}{ }^{\prime} \frac{d T \prime}{d t}
$$

A few things about Equation (2-8) should be noted. First, the apostrophe next to certain variables in the equation above indicates that the variable is mass-averaged throughout the reaction chamber. Also, chemical reaction does not occur in the MZM. The change in moles with respect to time term comes from mass flow from the reaction chamber to the crevice. The change rate of change in volume term represents the actual volume of the reaction chamber, and is derived from a piston position or velocity profile. Lastly, this equation assumes ideal gas. Equation 2-8 can be rearranged as follows:

$$
\frac{d T^{\prime}}{d t}=\frac{1}{N c_{v^{\prime}}}\left[\dot{Q}-P \frac{d V}{d t}+R T^{\prime} \frac{d N}{d t}\right]
$$

Using the differential form of the ideal gas equation (2-10), the pressure profile for the MZM can be calculated [S.S. Goldsborough, Mittal, \& Banyon, 2013]:

$$
\begin{aligned}
& \frac{1}{P} \frac{d P}{d t}+\frac{1}{V} \frac{d V}{d t}=\frac{1}{N} \frac{d N}{d t}+\frac{1}{T} \frac{d T^{\prime}}{d t} \\
& \frac{d P}{d t}=\frac{\gamma^{\prime} P}{N} \frac{d N}{d t}-\frac{\gamma^{\prime} P}{V} \frac{d V}{d t}+\frac{\gamma^{\prime}-1}{V} \dot{Q}
\end{aligned}
$$

The rate of pressure change from Equation (2-11) is applied to Equation (2-7), in order to find the effective volume profile of the HRM. 


\subsubsection{Multi-Stage Ignition Modeling}

One disadvantage of deriving volume profiles for HRM simulations from simulated or experimental non-reactive pressure traces is that the effects of multi-stage ignition cannot be directly accounted for. Multi-stage ignition in RCM experiments occurs due to the negative temperature coefficient (NTC) region, which initiates a preliminary set of chain branching reactions at lower temperatures. As temperature increases due to this preliminary combustion, the NTC region is exceeded, and chain branching reactions characteristic of higher temperatures become dominant, while NTC characteristic reactions cease [Curran, Gaffuri, Pitz, \& Westbrook, 1998].

In multi-stage ignition cases, first stage ignition increases the temperature in the reaction chamber, which increases the thermal gradient between the reactive mixture and the boundary, increasing heat loss. Obviously, non-reactive experiments or simulations cannot account for this effect. Therefore, HRM simulations that use a non-reactive pressure trace to calculate an effective volume profile will under-predict total heat loss, which will result in faster ignition times relative to RCM experiments or CFD simulations. First stage ignition can have additional multi-dimensional effects due to the presence of the boundary layer and crevice [Mittal, Raju, \& Sung, 2010]. For example, heat release rates may vary spatially within the thermal boundary layer, due to temperature variation. Another example is that increased pressure due to heat release in the reaction chamber could increase the pressure gradient between the reaction chamber and the crevice, inducing mass transfer between the two regions.

In a separate paper from which the original MZM was presented, Goldsborough et al. proposed two methods for accounting for multi-stage ignition within the MZM [S.S. 
Goldsborough et al., 2013]. The first method involves additional compression of the MZM mesh in response to heat release within the HRM. Compression is prescribed according to Equation 2-12:

$$
\frac{d V}{d t}=-\frac{V}{\gamma^{\prime}}\left[\left.\frac{1}{P} \frac{d P}{d t}\right|_{H R M}-\frac{\gamma^{\prime}}{N} \frac{d N}{d t}-\frac{\dot{Q}}{N c_{v^{\prime}}}\right]
$$

Equation 2-12 is a rearrangement of Equation 2-11 with the pressure rate of change of the MZM term replaced by the pressure rate of change of the HRM. Notice that when $\left.\frac{d P}{d t}\right|_{H R M}$ is equal to $\left.\frac{d P}{d t}\right|_{M Z M}$ (i.e. no chemical heat release in the HRM), then the volumetric compression of the MZM is the same as compression prescribed from the piston trajectory.

The second method for accounting for multi-stage ignition effects within the MZM is to add an additional heat addition term to the energy equation of the MZM reaction chamber zones in response to heat release in the HRM. Heat addition is calculated as follows:

$$
\dot{Q}_{a d d}=\frac{V}{\gamma^{\prime}-1}\left[\left.\frac{d P}{d t}\right|_{H R M}-\left.\frac{d P}{d t}\right|_{M Z M}\right]
$$

As in additional compression equation (2-12), Equation 2-13 is derived from Equation 111. The formulation of this equation assumes that the mole rate of change $\left(\frac{d N}{d t}\right)$ is negligible for first stage ignition. Both of the methods outlined above were applied to two cases of MZM simulations of n-heptane combustion [S.S. Goldsborough et al., 2013]. For both cases, it was shown that the heat addition method produced better agreement with CFD simulations in terms of pressure trace and ignition delay than the additional compression method. However, both methods still account for heat addition 
indirectly through pressure values in the HRM. The effects of chemical reaction outside the core region are neglected.

\subsubsection{New MZM Model}

This thesis presents a new MZM modelling approach that is based off the MZM of Goldsborough et al. [S. Scott Goldsborough et al., 2012] for RCM experiments. The new MZM approach models the effects of boundary work and conduction within the reaction chamber, as well as mass transfer from the reaction chamber to the crevice, just as the original model. However, the new MZM does not utilize an HRM. Rather, the computational zones that compose the reaction chamber of the RCM serve as reactors themselves.

There are many advantages to this modeling approach. First, it eliminates the need for a second reactor model. The model consists of just the MZM. Second, the effects of multi-stage ignition, which greatly affect ignition delay time and pressure traces during an RCM experiment, are directly accounted for since the computational zones within the MZM simulate chemistry. Last, this approach accounts for the effects of chemical reaction that occur outside of the core region, as opposed to the HRM, which represents the core region only. This is significant for several reasons.

For one, chemical heat release within the boundary layer, especially if the boundary layer temperature is within the NTC region, can be non-negligible and have significant effects on thermal stratification within the reaction chamber [Griffiths et al., 2001]. Second, it provides an advantage over the traditional HRM approach in predicting chemical species concentrations. Chemical speciation data within an HRM zone does not reflect average conditions within the entire RCM [Mittal, Raju, \& Sung, 2008]. Again, 
this is because the HRM simulates conditions in the core region only. The ability of the MZM to accurately predict species concentrations is important for validating mechanisms based on speciation data, which is a more rigorous metric for validation than ignition delay alone, as described earlier.

Details regarding the MZM are provided in the following chapter. This chapter describes how the reaction chamber mesh is formulated, as well as the various energy interactions that can occur for a computational zone. The reaction chamber, gap and crevice models are explained in detail as well. 


\section{Chapter 3 Multi-zone Model Description}

\subsection{Overview}

The outline of a computationally efficient multi-zone model (MZM) for the prediction of time dependent conditions within an RCM during experimentation is presented in this chapter. This model is an extension of the model presented by Goldsborough et al. [S. Scott Goldsborough et al., 2012], and has been reformulated to simulate chemistry throughout the entire reaction chamber of an RCM, as opposed to simulating chemistry within an "adiabatic core" region only. Simulating chemistry throughout the entire reaction chamber is essential for achieving accurate predictions of species concentrations, since reaction rates throughout the chamber spatially vary due to the thermal boundary layer. Speciation data is important because it provides a rigorous benchmark for evaluating chemical models and provides insight with regard to reaction pathways.

The MZM is composed of three separate sub-models which represent the reaction chamber, tapered gap, and crevice, respectively. No homogeneous reactor model (HRM) is included. Rather, chemical reaction is simulated in the MZM itself. The reaction chamber sub-model is comprised of a series of cylindrical, concentric zones whose total volume represents the volume of the reaction chamber at a given time. Each zone has unique and uniform properties (temperature, pressure, composition, etc.), as well as its own energy balance consisting of boundary work, conduction, enthalpy loss, and chemical heat source/sink terms. 
Volumetric compression of each zone occurs in response to piston movement predicted by a given RCM piston trajectory. Conduction between neighboring zones is modeled according to Fourier's law. An enthalpy loss term is also employed for each zone, which accounts for mass transfer from the chamber to the crevice.

Mass flows from the reaction chamber to the crevice via the tapered gap. It is assumed that mass flow through the tapered gap is quasi-static. Mass, momentum, and energy balances are employed in order determine the tapered gap inlet and exit velocity, as well as exiting temperature, assuming that the conditions at the inlet are known and can be calculated using the conditions within the reaction chamber. Flow through the tapered gap is driven by the pressure imbalance between the chamber and the crevice. This is reflected in the momentum equation. Energy from the tapered gap flow is lost to the boundaries via convective heat transfer, where the magnitude of heat loss is determined Reynolds and Nusselt number correlations to determine the heat transfer coefficient.

The crevice is modeled as an unsteady system whose state changes in response to convective heat transfer to the boundary and inlet mass flow from the tapered gap. Mass, momentum, and energy balances are again employed in order to determine the state in the crevice. Again, as in the tapered gap, Reynolds and Nusselt number correlations are used to determine the magnitude of the heat loss.

The remainder of this chapter is dedicated to expanding upon the above descriptions of each sub-model in greater detail. In the first sub-section, the reaction chamber model is discussed. The technique used to generate the grid is given, as well as 
a formulation of the energy balance for each zone. The remaining two sub-sections discuss the tapered gap and crevice models, respectively.

\subsection{Reaction Chamber Model}

\subsubsection{Mesh}

A series of concentric, cylindrical zones comprise the reaction chamber volume mesh in the MZM model. The innermost, or core zone is shaped as a solid cylinder, while the remaining zones are shaped as hollow cylinders with a uniform thickness. Each outside zone has uniform thickness, or in other words, the thickness of the upper and lower portions of the each zone is equal to its thickness in the radial direction. Constraining the size of each zone in such a way is essential for simplifying the calculation of conductive heat transfer between neighboring zones, which will become apparent in the next sub-section.

For a typical RCM experiment or simulation, large thermal gradients exist near the boundaries, due to the reaction chamber walls and piston having a significantly lower temperature than the compressed reaction chamber mixture. In order to resolve the thermal gradient and accurately model the conditions and conductive heat transfer within the boundary layer, greater grid refinement is applied near the boundaries of the reaction chamber. This is accomplished using a polynomial equation that determines the thickness of each zone in the reaction chamber mesh at the beginning of a simulation, based on the number of zones and the specified thickness of the outermost zone:

$$
\frac{B}{2}=t_{o}\left(1+\alpha+\alpha^{2}+\alpha^{3}+\cdots+\alpha^{z-1}\right)
$$


In this equation, $B$ is the bore of the reaction chamber, $t_{o}$ is the thickness of the outermost zone, $\alpha$ is the factor by which the thickness of each zone grows with respect its outside neighboring zone, and $z$ is the number of zones. The growth factor $\alpha$ is determined using a root finding algorithm. Figure 3-1 illustrates this grid generation technique.

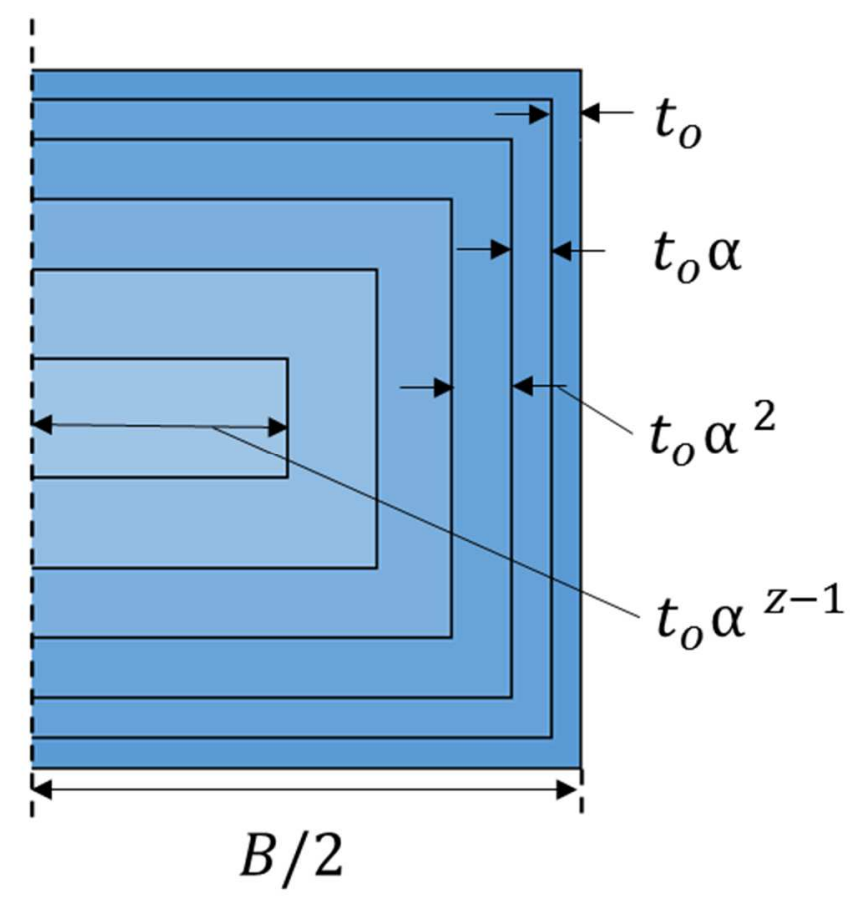

Figure 3-1: A 1-dimensional grid representation of the reaction chamber of an MZM, sliced along the axis of symmetry. The thickness of each zone increases by a factor of $\alpha$ with respect to its neighboring zone on the outside. Note, the sizes in this illustration are not necessarily to scale.

It should be noted that although the zones collectively represent a 3-dimensional reaction chamber, the mesh can be considered one-dimensional. This is because the conditions within a particular zone are uniform, and a zone directly interacts with neighboring zones only via conductive heat transfer. Also, as previously mentioned, the thickness of a zone is uniform, which means that a single value for the thermal gradient 
distance between two zones can be employed. Therefore, conductive heat transfer between zones can be described as a single interaction, and the series of interactions for every zone acting in a single dimension.

\subsubsection{Energy Formulation}

\subsubsection{Energy Equation for a Single Zone}

In the MZM, the reaction chamber is modeled as a thermally and compositionally nonhomogeneous mixture. The zones that comprise the reaction chamber, however, are homogeneous and exist at a single state. It is assumed that the reaction chamber mixture is an ideal gas, allowing the total internal energy of each zone to be defined accordingly:

$$
U=m \sum_{j} Y_{j} u_{j}(T)
$$

In Equation 3-2, variable $U$ represents the total internal energy of the system, which is a single zone in this case. Variable $m$ represents total mass, $Y$ is mass fraction, $u$ is internal energy, and subscript $j$ represents a specific species. It is important to note that the specific internal energy of a species $\left(u_{j}\right)$ is a function of temperature only, due to the ideal gas assumption. Equation 3-2 can be differentiated as follows:

$$
\frac{d U}{d t}=u \frac{d m}{d t}+m c_{v} \frac{d T}{d t}+m \sum_{j} u_{j} \frac{d Y_{j}}{d t}
$$

Neglecting the effects of kinetic and potential energy, the total internal energy of a zone can also be written as an energy balance:

$$
\frac{d U}{d t}=-P \frac{d V}{d t}+\dot{Q}-h \dot{m}_{o u t}
$$

The variable $\dot{Q}$ is the rate of heat transfer of a zone, $h$ is mass specific enthalpy and $\dot{m}_{\text {out }}$ is outward mass flow rate. 
Several assumptions are used to formulate the energy balance above (3-4). First of all, it is assumed that the presence of the crevice effectively suppresses the "roll-up" vortex in the reaction chamber, as discussed in Chapter 2. Therefore, advection within the reaction chamber is minimized, and it can be assumed that mass transfer between zones does not occur. In the current version of the model, it is also assumed that mass does not transfer from the crevice to the reaction chamber. The crevice and gap geometry of the RCCEM at Marquette University, which is the geometry used in the simulations in this thesis, is based off a study that used CFD to find an optimal geometry. Specifically, the gap and crevice geometry were optimized in order to swallow the shearing boundary layer fluid, and prevent its reemergence into the reaction chamber [Mittal \& Sung, 2007]. Therefore, assuming mass only transfers from the reaction chamber to the crevice is satisfactory. Since mass can only transfer out of a zone, the enthalpy inlet term has been omitted. It should also be noted that mass transfer to the crevice occurs for all zones, as opposed to the boundary layer zones only. This assumption is made in order to be consistent with a previous CFD study that have shown that high temperature gases enter the gap [Mittal et al., 2010]. Therefore Equation 3-4 is valid for every zone in the reaction chamber mesh.

Equations 3-4 and 3-5 can be combined to yield the following:

$$
m c_{v} \frac{d T}{d t}=-P \frac{d V}{d t}+\dot{Q}-h \dot{m}_{\text {out }}-u \frac{d m}{d t}-m \sum_{j} u_{j} \frac{d Y_{j}}{d t}
$$

Consider that for a reaction chamber zone the inlet mass flow rate is zero. Therefore, $\frac{d m}{d t}=-m_{\text {out }}$. Also consider that $h-u=\frac{P V}{m}$, where $m$ is the total mass in a zone. With these two considerations, Equation 3-5 can be rewritten as follows ["Reactor Networks," 2012]: 


$$
m c_{v} \frac{d T}{d t}=-P \frac{d V}{d t}+\dot{Q}-\frac{P V}{m} \dot{m}_{o u t}-m \sum_{j} u_{j} \frac{d Y_{j}}{d t}
$$

This form of the energy equation is attractive because it maximizes the use of primitive variables. The value of the last term, which can be thought of as the contribution of chemical reaction to the energy of the system, is calculated using a chemical kinetic mechanism. In fact, the last term is equal to $\sum_{j} V \dot{\omega}_{j} W_{j} u_{j}$, where $W_{j}$ is the molecular weight of species, and $\dot{\omega}_{j}$ is the rate of species production/destruction per unit volume. The rate of species evolution per unit volume is given from Equation 1-3 and is calculated using specific rate constants given from a mechanism. Knowing the current state of a reaction chamber zone, as well as the volume rate of change, heat transfer rate, and outward mass flow rate, Equation 3-6 can easily be integrated in order to obtain the temperature of a zone at a future time. From there, the future state of the zone can be easily defined. Methods for obtaining volume rate of change and heat transfer rate are discussed next. Discussion of the outward mass flow rate will be saved for the section that describes the tapered gap model, since flow through the tapered gap ultimately decides the mass flow rate from each reaction chamber zone. Figure 3-2 provides an illustration of the energy equation applied to a zone within the reaction chamber mesh of an MZM. 


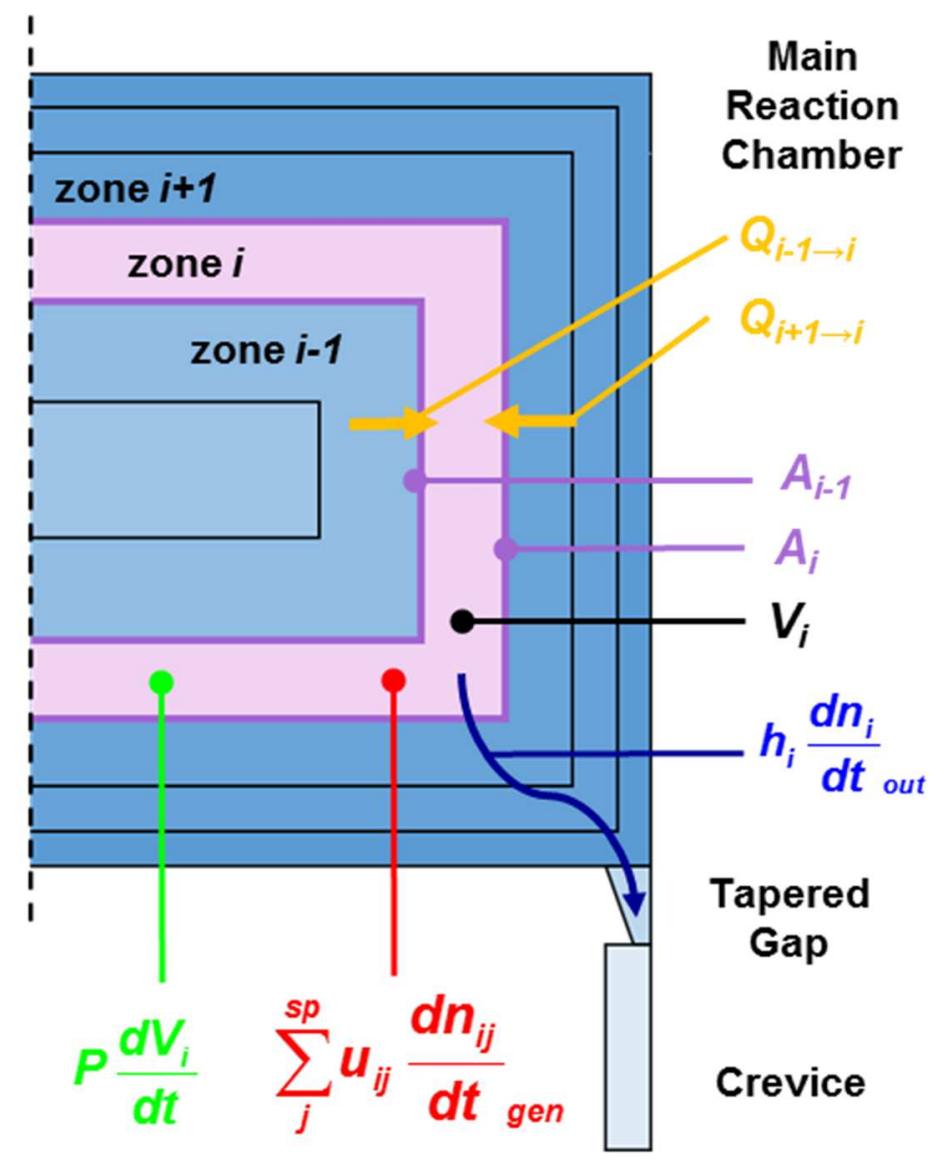

Figure 3-2: A 1-dimensional illustration of an MZM grid with 6 reaction chamber zones, sliced along the axis of symmetry. This figure highlights the different energy interactions that can occur within a single zone. The yellow text depicts conduction to/from neighboring zones. Boundary work and enthalpy loss to the crevice are represented by the green and blue text, respectively. Energy release due to chemical reaction is shown in red. Note, the sizes in this illustration are not necessarily to scale.

\subsubsection{Boundary Work}

The volume rate of change of the entire reaction chamber mesh is prescribed using an RCM piston velocity profile, which gives velocity of the piston with respect to time:

$$
\frac{d V_{R C}}{d t}=-\frac{\pi}{4} B^{2} v_{\text {piston }}(t)
$$


Here, $V_{R C}$ represents the total volume of the reaction chamber, while $B$ is the bore of the reaction chamber, and $v_{\text {piston }}$ is the velocity of the piston, which is a function of time. Since zone size within the reaction chamber domain varies, each zone is compressed according to the ratio of its individual volume with that of the total reaction chamber. As such, the boundary work of an individual zone is calculated accordingly:

$$
-P_{i} \frac{d V_{i}}{d t}=-\frac{n_{i} R T_{i}}{V_{i}} \frac{V_{i}}{V_{R C}} \frac{d V_{R C}}{d t}=-\frac{n_{i} R T_{i}}{V_{R C}} \frac{d V_{R C}}{d t}
$$

Here, the subscript $i$ represents a single zone, while $n$ represents the number of moles.

\subsubsection{Conductive Heat Transfer}

Heat transfer between neighboring zones occurs via conduction only. Convective heat transfer is assumed to be negligible since, as discussed earlier, the presence of the crevice minimizes advection within the reaction chamber. This assumption may not be good for conditions within the boundary layer, since it is known that boundary layer gas shears along the reaction chamber walls. However, this assumption simplifies the analysis, so it is used here. Conductive heat transfer for a particular zone is calculated using Fourier's law:

$$
\begin{gathered}
\dot{Q}_{i-1 \rightarrow i}=k_{i-1, i} A_{i-1} \frac{T_{i-1}-T_{i}}{\Delta x_{i-1 \rightarrow i}} \\
\dot{Q}_{i+1 \rightarrow i}=k_{i+1, i} A_{i} \frac{T_{i+1}-T_{i}}{\Delta x_{i+1 \rightarrow i}}
\end{gathered}
$$

Equations 3-9 and 3-10 describe heat transfer into a zone from its inside and outside neighbor, respectively. The variable $k$ is the thermal conductivity, which is determined using average temperature and composition between the two zones involved in heat transfer. Area at the border of the two zones exchanging heat is represented by $A$, while the thermal gradient distance is $\Delta x$. In general, the thermal gradient distance is taken to 
be the sum of the thicknesses of the zones involved in heat transfer, divided by two.

Conductive heat transfer for a zone is illustrated in Figure 3-3.

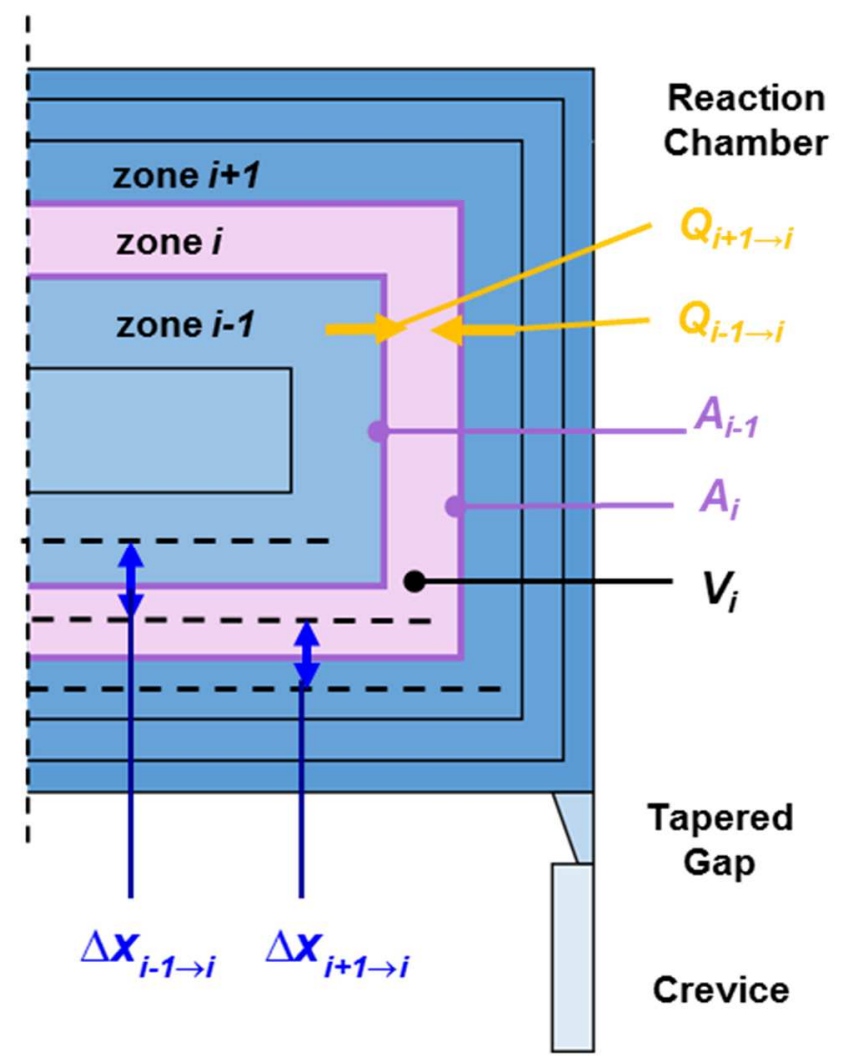

Figure 3-3: Illustration of conductive heat transfer for an MZM zone. Sizes in this illustration are not necessarily to scale.

For heat transfer from the outermost zone to the boundary, the thermal gradient distance is taken as half the thickness of the outermost zone. The thermal gradient distance for heat transfer from the core zone to the second innermost zone is assumed to be the radius of the core zone, plus half the thickness of the second innermost zone. As can be seen from Figure 3-3, the thickness of each zone must be uniform (thickness of the upper and lower portion of the zone must be equal to the radial thickness) in order for Equations 3-9 and 3-10 to be valid. If not, then the thermal gradient distance is inconsistent across the heat transfer area. 


\subsubsection{Cell Rezoning}

In the MZM, it is assumed that compression is quasi-static and that pressure is uniform throughout the reaction chamber at each time step. However, compressing each zone in the manner outlined above does not guarantee that pressure will be equal across all zones. Therefore, since no mass is transferred between reaction chamber zones, pressure equilibration is achieved through isentropic expansion/contraction of each zone until the pressure in the zone is equal to the average pressure across all zones. This process is performed at every time step. Zone volume and mass specific internal energy are modified during isentropic expansion/contraction as follows:

$$
\begin{aligned}
& V_{i, r}=V_{i}\left(\frac{P_{i}}{P_{R C}}\right)^{\frac{1}{\gamma_{i}}} \\
& u_{i, r}=u_{i}-\frac{1}{2}\left(P_{i}+P_{R C}\right) \frac{V_{i, r}-V_{i}}{m_{i}}
\end{aligned}
$$

Here, $P_{R C}$ refers to volume-averaged pressure across the reaction chamber, and subscript $r$ represents the condition after cell rezoning. After cell rezoning, the new temperature of the zone can either be inferred from the new internal energy, or calculated from an isentropic relation as well:

$$
T_{i, r}=T_{i}\left(\frac{V_{i}}{V_{i, r}}\right)^{\gamma_{i}-1}
$$

The height and radius of each zone after resizing must be calculated. Each zone must maintain uniform thickness after resizing in order to enforce a uniform thermal gradient distance. This criteria is satisfied by requiring that the magnitude of growth/shrinkage of the outer height of a zone must be double the growth/shrinkage of the outer radius. In other words, the distance by which each edge moves must be equal. 
That way, the uniform thickness across the zone that was present before resizing is preserved. The height/radius of the inside of a particular zone is determined by the outside height/radius of the inside neighbor of the zone. The magnitude by which the outer surface of a particular zone grows/shrinks $(\beta)$ can be described mathematically as follows:

$$
\sum_{y=0 . i} V_{y, r}=\pi\left(r_{i, \text { out }}+\beta\right)^{2}\left(h_{i, \text { out }}+2 \beta\right)
$$

In Equation 3-14, the term on the left-hand side represents the sum of the volumes of the zone of interest, and all zones on the inside of the zone of interest. Subscript 0 represents the core zone, while subscript $i$ represents the zone of interest. Variables $r_{i, \text { out }}$ and $h_{i, \text { out }}$ represent the outside radius and height of the zone of interest, respectively. Since the volumes after resizing, and the initial radii and heights of each zone are known, a root finding algorithm is used to determine $\beta$ from Equation 3-14. Figure 3-4 illustrates the zone resizing technique.

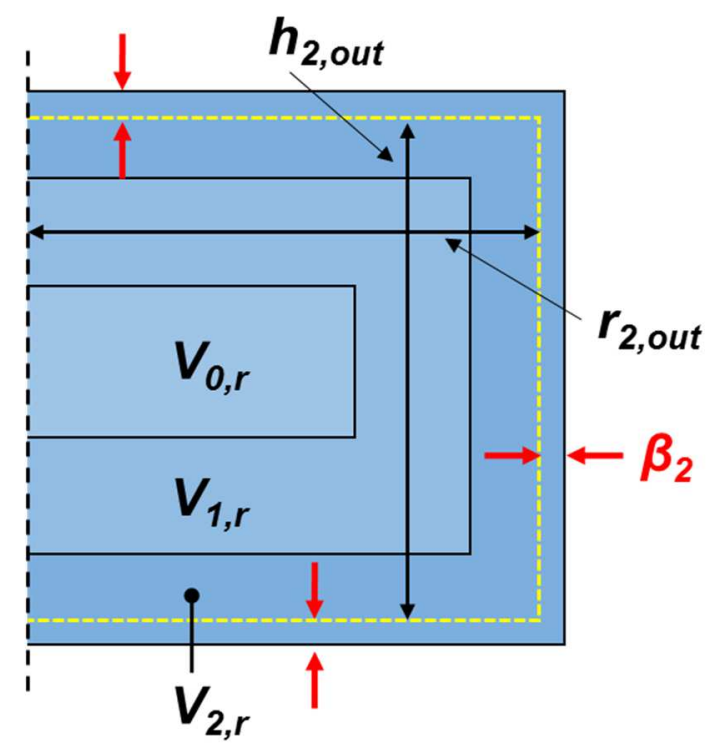

Figure 3-4: Example illustration of the cell rezoning technique for the third innermost zone in an MZM reaction chamber mesh. The outer radius and height of the zone before 
resizing, denoted by $r_{2, \text { out }}$ and $h_{2, \text { out }}$, respectively, must increase by $\beta_{2}$ and $2 \beta_{2}$ respectively, in order to achieve the resized volume.

\subsection{Tapered Gap Model}

\subsubsection{Governing Equations}

Reaction chamber gas is assumed to flow quasi-steadily through the tapered gap and then into the crevice. This means that the mass, momentum, and energy entering the gap at a given time are equal to the mass, momentum, and energy exiting respectively. The quasi-steady assumption is advantageous because it allows for the tapered gap mass flow and exiting conditions to be easily solved for, both of which ultimately affect the conditions within the reaction chamber. It is also assumed that mass flow through the gap is induced by the pressure gradient between the reaction chamber and the crevice. This is apparent in the momentum equation. Mass, momentum, and energy balances for the tapered gap can be written accordingly:

$$
\begin{aligned}
& \rho_{\text {gap }, \text { in }} A_{\text {gap }, \text { in }} v_{\text {gap }, \text { in }}-\rho_{\text {gap }, \text { ex }} A_{\text {gap }, e x} v_{\text {gap }, e x}=0 \\
& \rho_{\text {gap }, \text { in }} A_{\text {gap }, \text { in }} v_{\text {gap }, \text { in }}{ }^{2}-\rho_{\text {gap }, e x} A_{\text {gap }, \text { ex }} v_{\text {gap }, e x}{ }^{2}-\tau_{\text {gap }} A_{\text {gap }, \text { surf }} \\
& +\left(P_{R C}-P_{\text {crev }}\right) A_{\text {gap }, e x}=0 \\
& q_{\text {gap }} A_{\text {gap }, \text { surf }} \\
& +\dot{m}_{\text {gap }, \text { in }}\left[\left(h_{\text {gap }, \text { in }}-h_{\text {gap }, e x}\right)+\frac{1}{2}\left(v_{\text {gap }, \text { in }}{ }^{2}-v_{\text {gap }, e x}{ }^{2}\right)\right]=0
\end{aligned}
$$

In the above equations, $\rho, A$ and $v$ are density, area and velocity respectively. The indices in, ex and surf refer to inlet and exit conditions of the crevice, respectively. Friction and convective heat transfer within the gap are represented by $\tau_{\text {gap }}$ and $q_{\text {gap }}$. 
Friction along the cylinder wall and piston head of the tapered gap flow is calculated using a mean friction coefficient. The mean friction coefficient is calculated using a correlation for developing flow in an annulus [S. Scott Goldsborough et al., 2012; Kays, Crawford, \& Weigand, 2004].

$$
\begin{aligned}
& \tau_{\text {gap }}=C_{f, \text { gap }}\left(0.5 \rho_{\text {gap }} v_{\text {gap }}{ }^{2}\right) \\
& C_{f, \text { gap }}=\frac{24}{R e_{\text {gap }}}\left[\frac{0.008\left(\zeta_{\text {gap }} / l_{\text {gap }}\right) R e_{\text {gap }}}{1+0.025\left[\left(\zeta_{\text {gap }} / l_{\text {gap }}\right) R e_{\text {gap }}\right]^{2 / 3}}\right]
\end{aligned}
$$

In the friction coefficient equation, $R e$ refers to Reynolds number, $l_{g a p}$ is the length of the gap in the axial direction, and $\zeta_{g a p}$ is the characteristic length of the gap, which in this case is the mean hydraulic diameter.

Due to the high gas velocities within the tapered gap, it is assumed that heat loss to the piston head and the cylinder wall occurs by convection only. Convective heat transfer is calculated using the log mean temperature difference equation to estimate the average temperature difference of the flow with the boundaries, and utilizing a Nusselt number equation for developing flow in an annulus in order to estimate the convection coefficient $\left(h_{\text {conv,gap }}\right)$ [S. Scott Goldsborough et al., 2012; Kays et al., 2004]:

$$
\begin{aligned}
& q_{\text {gap }}=h_{\text {conv,gap }}\left(T_{\text {gap }, e x}-T_{\text {gap }, \text { in }}\right) \ln \left[\frac{T_{\text {gap }, e x}-T_{\text {gap }, \text { in }}}{T_{\text {gap }, e x}-T_{\text {wall }}}\right] \\
& N u_{\text {gap }}=\frac{h_{\text {conv,gap }} \zeta_{\text {gap }}}{k_{\text {gap }}}=7.54+\frac{0.028\left(\zeta_{\text {gap }} / l_{\text {gap }}\right) R e_{\text {gap }} P r_{\text {gap }}}{1+0.011\left[\left(\zeta_{\text {gap }} / l_{\text {gap }}\right) R e_{\text {gap }} P r_{\text {gap }}\right]^{2 / 3}}
\end{aligned}
$$

Here, $P r_{g a p}$ refers to Prandtl number, and $k_{\text {gap }}$ represents thermal conductivity of the gap mixture.

Since prior CFD work has shown that gas at elevated temperatures as well as boundary layer gas enters the gap, mass is assumed to flow evenly from all zones into the 
tapered gap. Therefore, the inlet temperature into the gap can be estimated as the average temperature between all reaction chamber zones, which is effectively the mass-averaged temperature of the inlet flow. Inlet molecular weight is calculated in a similar fashion. Mole fraction of each species at the inlet is determined by calculating the total number of moles that enter the gap if mass flow from each zone is identical, and determining what fraction of the moles are the species of interest. Inlet pressure is assumed to be the same as the reaction chamber pressure.

\subsubsection{Newton's Method}

With the thermodynamic state at the gap inlet known, the only unknowns left in the mass, momentum and energy equations of the gap are inlet and exit velocity, and exit temperature. However, due to the high non-linearity of the mass, momentum, and energy equations with respect to the unknown variables, achieving an algebraic solution of the unknowns is unfeasible. Rather, Newton's method is used to converge upon estimates of the unknown variables.

The underlying principle behind Newton's method is that if a function and its first derivative are known at a specific point $\left(x_{0}\right)$, then the value of the function at a new point $(x)$ can be estimated. This can be written as follows:

$$
f(x) \approx f\left(x_{0}\right)+f^{\prime}\left(x_{0}\right)\left(x-x_{0}\right)
$$

Equation 3-22 is valid for multiple functions that share independent variables. In this case, each term in the equation is a vector. For example, consider a system with three functions and three variables of interest, such as the system of tapered gap equations. The vector $x_{0}$ contains the values of the independent variables for which the values of the functions are known, say $\left(x_{1,0}, x_{2,0}, x_{3,0}\right)$, while the vector $x$ contains the new values for 
the independent variables $\left(x_{1}, x_{2}, x_{3}\right)$. With $x_{0}$ and $x$ defined as above, the linear estimation of the collection of functions at new values of the independent variables can be written as follows:

$$
\left[\begin{array}{l}
f_{1}(x) \\
f_{2}(x) \\
f_{3}(x)
\end{array}\right] \approx\left[\begin{array}{l}
f_{1}\left(x_{0}\right) \\
f_{2}\left(x_{0}\right) \\
f_{3}\left(x_{0}\right)
\end{array}\right]+\left[\begin{array}{lll}
\frac{\partial f_{1}}{\partial x_{1}}\left(x_{0}\right) & \frac{\partial f_{1}}{\partial x_{2}}\left(x_{0}\right) & \frac{\partial f_{1}}{\partial x_{3}}\left(x_{0}\right) \\
\frac{\partial f_{2}}{\partial x_{1}}\left(x_{0}\right) & \frac{\partial f_{2}}{\partial x_{2}}\left(x_{0}\right) & \frac{\partial f_{2}}{\partial x_{3}}\left(x_{0}\right) \\
\frac{\partial f_{3}}{\partial x_{1}}\left(x_{0}\right) & \frac{\partial f_{3}}{\partial x_{2}}\left(x_{0}\right) & \frac{\partial f_{3}}{\partial x_{3}}\left(x_{0}\right)
\end{array}\right]\left[\begin{array}{l}
x_{1}-x_{1,0} \\
x_{2}-x_{2,0} \\
x_{3}-x_{3,0}
\end{array}\right]
$$

It should be firstly noted that in order to use Newton's method, each function must be equal to zero. In the case of the tapered gap, this requirement is satisfied due to the quasi-steady assumption. Hence, the quasi-steady assumption is necessary for finding the unknowns using Newton's method. Since each function is equal to zero, it is desired to find $x$ such that $f(x)$ is zero:

$$
f\left(x_{0}\right)+f^{\prime}\left(x_{0}\right)\left(x-x_{0}\right)=0
$$

Since $f^{\prime}\left(x_{0}\right)$ is a square matrix, its inverse can be calculated. As a result, Equation 3-24 can be arranged in order to yield $x$ :

$$
x=x_{0}-\left(f^{\prime}\left(x_{0}\right)\right)^{-1} f\left(x_{0}\right)
$$

Since Equation 3-25 is only a linear approximation of what value of $x$ satisfies the functions, evaluation of Equation 3-25 is performed for many iterations. At each iteration, $x_{0}$ is updated from to be equal to $x$ from the previous iteration. The iterative evaluations of Equation 3-25 continue until the difference between $x$ and $x_{0}$ is small enough according to a convergence criteria.

Again, since the mass, momentum and energy equations are highly nonlinear with respect to the velocities and outlet temperature, the derivatives in the derivative matrix $f^{\prime}\left(x_{0}\right)$ cannot be determined analytically. Each of the nine derivatives in the matrix are 
estimated using a central difference scheme. An example central difference calculation for $\frac{\partial f_{1}}{\partial x_{2}}$ is provided below:

$$
\frac{\partial f_{1}}{\partial x_{2}}=\frac{f_{1}\left(x_{1}, x_{2}+\Delta x_{2}, x_{3}\right)-f_{1}\left(x_{1}, x_{2}-\Delta x_{2}, x_{3}\right)}{2 \Delta x_{2}}
$$

Equation 3-26 is calculated for every combination of function (mass, momentum, and energy balance) and unknown independent variable (inlet and outlet velocity, and outlet temperature. For the two functions in the denominator, only the variable for which the derivative is with respect to is varied. The other variables are kept at the current guess.

\subsection{Crevice Model}

\subsubsection{Governing Equations}

The crevice in the MZM is assumed to be a single-zone volume that is not in thermal equilibrium with the cylinder wall and piston. Chemical reaction within the crevice is neglected. The crevice receives mass flow from the reaction chamber via the tapered gap, and therefore must be modeled as an unsteady system. Also, since there is a thermal gradient between the crevice and the cylinder wall and piston, heat transfer takes place. It should be noted that the formulation here assumes that no mass escapes from the crevice into the piston ringpack region. In a real RCM experiment, some leakage may occur, which may slightly alter the pressure within the crevice and even the reaction chamber. This phenomenon is modeled in the original MZM. However, since the ultimate goal of the study is to determine the adequacy of modeling the reaction chamber zones as reactors in an MZM, and since the adequacy of this approach is evaluated by comparing speciation data with CFD simulations, which make the same simplifying assumption, this assumption can be made. Also, accounting for blow-by in the original 
MZM for cases involving hydrogen combustion resulted in only minimal differences in reaction chamber pressure compared to neglecting blow-by, especially at moderate pressures. At 30 bar, blow-by only affected the reaction chamber pressure by $0.2 \%$ to $1 \%$ from $20 \mathrm{~ms}$ to $100 \mathrm{~ms}$ after TDC[S. Scott Goldsborough et al., 2012].

The mass, momentum, and energy formulations for the crevice zone are written as follows:

$$
\begin{aligned}
& \dot{m}_{g a p, e x}=\frac{d m_{\text {crev }}}{d t} \\
& \rho_{\text {gap }, e x} A_{\text {gap }, e x} v_{\text {gap }, e x}{ }^{2}-0.5\left(\tau_{\text {wall }}+\tau_{\text {pist }}\right) A_{\text {crev }, \text { surf }}=\frac{d}{d t}(m v)_{c r e v} \\
& q_{c r e v} A_{c r e v, \text { surf }}+\dot{m}_{\text {gap }, \text { ex }}\left[h_{\text {gap }, e x}+\frac{1}{2} v_{g a p, e x}{ }^{2}\right]=\frac{d}{d t}(m u)_{c r e v}
\end{aligned}
$$

The nomenclature used in the crevice equations are similar to that of the tapered gap equations. Subscript crev represents the crevice. Friction occurs within the flow exiting the gap and traveling along the cylinder wall, represented by $\tau_{\text {wall }}$, and within the recirculating flow along the bottom of the crevice and along the piston, represented by $\tau_{\text {pist }}$. Flow exiting the gap is assumed to behave as a jet, and therefore, the friction coefficient and friction are modeled as such [S. Scott Goldsborough et al., 2012; Villafruela, Castro, \& Parra, 2008]:

$$
\begin{aligned}
& \tau_{\text {wall }}=C_{f, j e t}\left(0.5 \rho_{\text {gap }, e x} v_{\text {gap }, e x}{ }^{2}\right) \\
& C_{f, j e t}=0.0042+0.0021 \log \left(R e_{\text {gap }, e x}\right)
\end{aligned}
$$

Friction and friction coefficient for the recirculating flow along the piston is calculated in a similar fashion as friction in the tapered gap:

$$
\begin{aligned}
& \tau_{\text {pist }}=C_{f, p i s t}\left(0.5 \rho_{\text {crev }} v_{\text {crev }}{ }^{2}\right) \\
& C_{f, p i s t}=\frac{24}{R e_{\text {crev }}}\left[\frac{0.008\left(\zeta_{\text {crev }} / l_{\text {crev }}\right) R e_{\text {crev }}}{1+0.025\left[\left(\zeta_{\text {crev }} / l_{\text {crev }}\right) R e_{\text {crev }}\right]^{2 / 3}}\right]
\end{aligned}
$$


Here, the characteristic length of the crevice $\left(\zeta_{\text {crev }}\right)$ is taken to be the ratio of crevice volume to surface area.

Heat transfer from the crevice volume to the boundaries is assumed to occur by convection only in the model. Convective heat loss is calculated by determining the convection coefficient, which is determined from a Nusselt number correlation, and multiplying by the temperature gradient between the crevice and wall.

$$
q_{\text {crev }}=h_{\text {conv }, \text { crev }}\left(T_{\text {wall }}-T_{\text {crev }}\right)
$$

Up until this point, each presented friction coefficient and Nusselt number correlation is consistent with the original MZM. However, the Nusselt number correlation and convection coefficient equations have been modified for the presented MZM accordingly:

$$
\begin{aligned}
& h_{\text {conv }, \text { crev }}=\frac{N u_{\text {crev }} k_{\text {crev }}}{\zeta_{\text {crev }}} \\
& N u_{\text {crev }}=11 P r_{\text {crev }}{ }^{-0.6}+\frac{0.028\left(\zeta_{\text {crev }} / l_{\text {crev }}\right) R e_{\text {crev }} P r_{c r e v}}{1+0.011\left[\left(\zeta_{\text {crev }} / l_{\text {crev }}\right) R e_{\text {crev }} P r_{\text {crev }}\right]^{2 / 3}}
\end{aligned}
$$

The Nusselt number correlation above is different from that of the original model in that the first term for the original model is a constant value (7.54) instead of a function of Prandtl number. In the original model, the convection coefficient is an average of the formula for convection coefficient shown above, and an additional term that's a function of friction factor and the Reynolds number of the gas jet exiting the tapered gap. Equation 3-35 is the fundamental definition of convective heat transfer coefficient. It is postulated that the effect of the gas jet exiting the gap does not need to be explicitly accounted for in the convection coefficient equation, since the gas jet clearly affects the velocity of the crevice as can be seen from observing the momentum equation. 
Using Equations 3-35 and 3-36, as opposed to the original equations for Nusselt number and convection coefficient, improved agreement between MZM and CFD simulation results for both hydrogen and primary reference fuel (PRF) combustion. Specifically, MZM simulations that utilize the original Nusselt number correlation and convection coefficient yield significantly shorter ignition delay times compared to CFD simulations. This is especially true for simulations with longer ignition delay times ( $\sim 60$ $100 \mathrm{~ms}$ ). It makes sense that the original correlations do not necessarily yield agreement with CFD results for the proposed modeling approach. The proposed modeling approach directly accounts for heat release outside the core and heat release before the main ignition event, while the original modeling approach accounted for pre-combustion heat release only, and did so by compressing the MZM mesh. With the effects of the crevice aside, predicted conditions within the reaction chamber are likely to be different for the two modeling approaches outlined above.

\subsubsection{Finite Difference Method}

The mass, momentum and energy equations that describe the crevice (Equations $3-27,3-28$, and 3-29) are discretized and integrated in order to determine the state of the crevice at the subsequent time step. Discretization of the mass equation using a forward difference is straightforward:

$$
\dot{m}_{g a p, e x, t}=\frac{m_{c r e v, t+\Delta t}-m_{c r e v, t}}{\Delta t}
$$

Above, subscript $t$ represents the current time, while $t+\Delta t$ is the time at the next time step. Equation 3-37 can be rearranged to yield the mass of the crevice at the subsequent time step, where $\dot{m}_{g a p, e x}$ is known and calculated from the tapered gap model:

$$
m_{\text {crev }, t+\Delta t}=\dot{m}_{g a p, e x, t} \Delta t+m_{c r e v, t}
$$


The momentum equation (3-38) can be expanded as follows:

$$
M=\frac{d}{d t}(m v)_{\text {crev }}=\frac{d m_{\text {crev }}}{d t} v_{\text {crev }}+\frac{d v_{\text {crev }}}{d t} m_{\text {crev }}
$$

Above, the symbol $M$ compactly represents the entire left-hand side of the momentum equation (3-28). The evaluation of $M$ is straight forward at a given time step, since each term on the left hand side of the momentum equation are functions of values at the present time step, which are known. Equation 3-39 can also be discretized using a forward difference:

$$
M=\dot{m}_{\text {gap }, e x, t} v_{\text {crev }, t}+\frac{v_{\text {crev }, t+\Delta t}-v_{\text {crev }, t}}{\Delta t} m_{\text {crev }, t}
$$

It should be noted that $\frac{d m_{\text {crev }}}{d t}$ in Equation 3-39 is replaced by $\dot{m}_{g a p, e x}$ in the discretized form above. With $M, \dot{m}_{g a p, e x, t}$, and the current mass and velocity known, Equation 3-40 can be manipulated in order to solve for velocity in the crevice at the subsequent time step:

$$
v_{\text {crev }, t+\Delta t}=\left(M-\dot{m}_{\text {gap }, e x, t} v_{\text {crev }, t}\right) \frac{\Delta t}{m_{\text {crev }, t}}+v_{\text {crev }, t}
$$

The energy equation of the crevice (3-29) can also be discretized in order to find the internal energy of the crevice gas at the next time step. This process for finding the new internal energy is very similar to that of finding the new velocity outlined above. Therefore, it will not be reviewed here.

With the mass and internal energy in the crevice known, the thermodynamic state of the crevice gas can be fixed. Crevice temperature at the subsequent time step can be calculated using the ideal gas relation for temperature with internal energy: $d T=\frac{u(T)}{c_{v}(T)}$. Crevice pressure, which plays a key role in determining mass transfer between the reaction chamber and crevice, is calculated using the ideal gas law. 


\subsection{Implementation}

The MZM as described above is coded using Python programming language for this particular study, while the chemistry equation solver software Cantera is used for determining chemical species at each time step, as well as solving the the mass and energy equations for the reaction chamber zones. At the beginning of the simulation the initial conditions (i.e. temperature, pressure and mole fraction of each species present) are set, as well as the overall geometry of the RCM (bore, gap width and length, crevice dimensions, etc.) defined. The geometry of the reaction chamber zones are then defined according to the grid generation equation discussed above (Equation 3-1). Input parameters determine number of zones and thickness of the outermost zone for this step. The mass and number of moles in each zone are calculated. Other conditions (temperature, pressure, specific internal energy, etc.) of each zone should be the same as the specified initial conditions.

The MZM steps through a series of iterations during a simulation, each of which represents a different time step. For each iteration, cell rezoning is calculated first, followed by conductive heat transfer, and then mass transfer from the reaction chamber to the crevice (from the tapered gap model). The chemical evolution rate equations and energy equation are then integrated for each reaction chamber zone. Finally, the crevice model is utilized to update conditions in the crevice. For the initial time step, cell rezoning, conductive heat transfer, and the tapered gap equations are essentially skipped, since there hasn't yet been any boundary work to induce a thermal gradient between zones or a pressure gradient between reaction chamber and crevice. Therefore, the 
discussion of MZM implementation will begin with compression and solving the energy equation.

Compression of the reaction chamber mesh is initiated according to a userprovided piston profile, which specifies the speed of the piston at a series of simulation times. A Cantera reactor is formulated for each reaction chamber zone, where the temperature, pressure, mole fractions, and volume of each zone are communicated to Cantera. The rate of volume change of each zone (determined according to the ratio of the volume of the zone with the entire reaction chamber volume) is also specified. Cantera then integrates the energy equation (3-6) as well as chemical species production rate equation (2-3), solving for temperature and chemical species concentrations/mole fractions. The volume and mass of each zone are known from the provided volume profile and the mass balance equation respectively, allowing the state in each zone to be updated. Calculations regarding the crevice, which come after the energy and chemical calculations for the reaction chamber zones, are essentially skipped for the first time iteration, since the tapered gap equations are yet to predict mass flow from the reaction chamber to the crevice.

The second iteration then begins. First, the overall or average conditions within the entire reaction chamber are determined from the conditions within each reaction chamber zone. Specifically, volume averaged pressure, mass averaged temperature, molecular weight and total mass are calculated. With the reaction chamber pressure known, the cell rezoning step, as described in section 3.2.2.4, is performed in order to equalize the pressure across all reaction chamber zones. The temperature, pressure, volume and internal energy of each zone are then updated accordingly. The magnitude of 
conductive heat transfer between neighboring zones and from the outermost zone to the wall is then calculated according to Equations 3-9 and 3-10. Thermal conductivity for each instance of heat transfer is calculated using the average temperature and species fractions between neighboring zones.

Mass loss from the reaction chamber zones is then calculated by utilizing the tapered gap model. First, the inlet conditions into the tapered gap are calculated as outlined above in section 3.3.1. Second, the initial guesses for each unknown variable in the tapered gap mass, momentum, and energy equations are specified. At the first time step in a simulation, the initial guesses for gap inlet and exit velocity are zero, while the initial guess for outlet temperature is the RCM temperature specified in the initial conditions. For the remaining time steps, the initial guesses of the unknowns are the converged upon values from the previous time step. Newton's method, as described in section 3.3.2, is then utilized in order to find the inlet and exit velocity, and therefore the mass flow from the reaction chamber.

A Cantera reactor is then formulated for each zone, and the energy and chemical equations are integrated, similar to procedure described paragraphs above. The only difference in formulating the Cantera reactor between the first and second iteration is that in the second iteration, outward mass flow and conduction heat transfer must also be specified, since these values are non-zero. After the energy and chemical evolution equations are integrated for each zone, the crevice model is used to update the conditions within the crevice. The third iteration then begins, and the simulation proceeds until a user-specified termination criterion is met. The typical termination criterion utilized for 
this work is a maximum reaction chamber temperature, which is exceeded during the main combustion event, effectively ending the simulation at the time of combustion. 


\section{Chapter 4 Multi-zone Model Validation}

The proposed MZM described in the previous chapter must be validated in order to ensure that it yields accurate predictions of conditions inside RCMs during experimentation. Specifically, the MZM should be able to accurately predict pressure and temperature profiles, since pressure is directly measured in RCM experiments, and temperature largely governs chemical reaction rates. Also, it is important that the MZM is able to predict species concentrations and mole fractions. The main motivation for reformulating the MZM so that each reaction chamber zone simulates the effects of chemical reaction is so that the MZM can be used for validating chemical models based on speciation data from RCM experiments with reaction quenching. Speciation data is a direct reflection of the reaction rates and reaction pathways that a chemical model is attempting to predict, and is therefore important for validation. Temperature, pressure, and species concentrations in an RCM are all interrelated. Therefore, the MZM should be able to predict all three.

Since the purpose of the MZM is to predict species concentrations in physical RCM experiments, an ideal method for validating the MZM is to perform quenching CFD experiments and compare the speciation results with results obtained from the MZM. For this method, the MZM would use a chemical model which has already been validated in the literature. However, quenching RCM experiments have not yet been done at Marquette, but are being planned for the near future.

Rather, the model is validated by comparing the results obtained from MZM simulations with results from CFD simulations for a range of conditions. As stated in Chapter 2, CFD is a rigorous method for numerically modeling conditions in the RCM, 
and directly accounts for heat loss and gas dynamic effects. Therefore, data from CFD simulations provide a good baseline for comparison with MZM data, and provide a way of assessing the accuracy of MZM simulations. The following sub-section describes the various test cases and their associated conditions for which MZM and CFD simulations are performed.

\subsection{Test Cases}

The MZM and CFD results presented in this thesis are from nine different cases, with each having a different combination of fuel type and compression ratio. Each test case has the same initial temperature $(300 \mathrm{~K})$ and compressed pressure ( $25 \mathrm{bar})$, and utilizes an identical piston trajectory. For the trajectory used for this study, the piston travels 8 inches in $32 \mathrm{~ms}$, achieving a maximum velocity and acceleration of approximately $19 \mathrm{~m} / \mathrm{s}$ and $2000 \mathrm{~m} / \mathrm{s}^{2}$, respectively. With stroke length fixed, compression ratios are varied by adjusting the clearance height between the piston and the head of the cylinder.

The simulated cases consist of three different fuels/fuel-blends, and three compression ratios for each fuel, totaling nine cases. Compression ratios for each fuel are chosen in order to yield a range of ignition delay times between 10 and $90 \mathrm{~ms}$ after compression is completed. Each simulation possesses a stoichiometric air to fuel ratio, with the three possible fuels being $n$-heptane, iso-octane, and a $50 \%$ mixture by volume of the two. The compressed pressure and initial mixture temperature for each simulation are $\sim 25$ bar and $300 \mathrm{~K}$, respectively. A constant cylinder wall and piston temperature of $300 \mathrm{~K}$ is assumed for each case. Compression ratios of 11,12 and 13 are used for the iso-octane cases. The compression ratios for the $n$-heptane and $n$-heptane/iso-octane 
simulations are 9, 10 and 11. An overview of the conditions for each case is presented in the table below. It should be noted that the compressed pressures and temperatures in the table come from the MZM results.

\begin{tabular}{|c|c|c|c|c|}
\hline Case & $\begin{array}{c}\text { Compression } \\
\text { Ratio }\end{array}$ & $\mathbf{P}_{\mathbf{c}}(\mathbf{b a r})$ & $\mathbf{T}_{\mathbf{c}}(\mathbf{K})$ & $\begin{array}{c}\text { Molar Composition } \\
\mathbf{O}_{2} / \mathbf{N}_{\mathbf{2}} / \mathbf{C}_{\mathbf{8}} \mathbf{H}_{\mathbf{1 8}} / \mathbf{C}_{\mathbf{7}} \mathbf{H}_{\mathbf{1 6}}\end{array}$ \\
\hline 1 & 11 & 24.4 & 651 & $0.2066 / 0.7769 / 0.0165 / 0.0$ \\
2 & 12 & 24.2 & 668 & $0.2066 / 0.7769 / 0.0165 / 0.0$ \\
3 & 13 & 24.0 & 683 & $0.2066 / 0.7769 / 0.0165 / 0.0$ \\
4 & 9 & 25.1 & 617 & $0.2062 / 0.7751 / 0.0 / 0.0187$ \\
5 & 10 & 25.0 & 636 & $0.2062 / 0.7751 / 0.0 / 0.0187$ \\
6 & 11 & 24.8 & 654 & $0.2062 / 0.7751 / 0.0 / 0.0187$ \\
7 & 9 & 25.1 & 616 & $0.2064 / 0.7760 / 0.0083 / 0.0093$ \\
8 & 10 & 24.9 & 635 & $0.2064 / 0.7760 / 0.0083 / 0.0093$ \\
9 & 11 & 24.8 & 653 & $0.2064 / 0.7760 / 0.0083 / 0.0093$ \\
\hline
\end{tabular}

Table 4-1: An overview of the conditions for each test case for which MZM and CFD simulations are performed. Shown for each case is the compressed temperature and pressure, compression ratio, and initial mole fractions

An initial temperature of $300 \mathrm{~K}$ is chosen for each case since the fuel/air mixture in RCM experiments is often in thermal equilibrium with the environment before compression. Although, it should be noted that there have been experiments where fuel/air mixture is heated in order to ensure that the fuel remains in vapor phase [Kumar, Mittal, \& Sung, 2009]. RCM experiments commonly produce compressed pressures between 10 to 40 bar. Therefore, a compressed pressure of $\sim 25$ bar is used for each simulation, since it is the middle ground between the two extremes. An initial pressure that yields a compressed pressure of 25 bar is estimated for each case according to the isentropic relationship between pressure and volume: 


$$
P_{0}=P_{c} C R^{\gamma}
$$

Here, $C R$ represents compression ratio. The ratio of specific heats $(\gamma)$ is estimated using pressure values and compression ratios from previous simulations with similar conditions, using the same equation above.

Compression ratios are varied in order to produce ranges of ignition delays between 15 and $90 \mathrm{~ms}$. Each fuel is chosen in order to test the MZM against a variety of ignition characteristics. For the conditions utilized for the simulations presented in this thesis, the combustion of n-heptane is single-stage, consisting of only one main heatrelease event. However, the combustion of iso-octane at the given conditions is considered two-stage, where a preliminary heat-release event commences and then quickly ceases due to reaction chamber gas temperatures entering and then exiting the NTC region. This is followed by the main heat-release event. For the $50 \% / 50 \%$ blend of n-heptane and iso-octane, two different stages of ignition are distinctly present. However, both ignition events occur very close together in time.

Conditions that yield two-stage ignition are specifically chosen for the MZM and CFD simulations, since accounting for heat loss during and after first-stage ignition has proven to be a weakness for past reduced-order modelling approaches. These approaches have either completely neglected the effects of preliminary heat release, or have only accounted for them indirectly. See section 2.3.3.2 for more details regarding methods for accounting for the effects of multi-stage ignition in reduced order models. Since the MZM presented in this thesis directly simulates chemistry throughout the entire reaction chamber, it is postulated that it does a better job of directly accounting for the effects of preliminary heat release before the main combustion event compared to other approaches. 
Simulation data from cases involving two-stage ignition is necessary for evaluating this postulation.

The chemical mechanism developed by Tsurushima for the combustion of primary reference fuels at HCCI conditions [Tsurushima, 2009] is utilized for calculating chemical evolution rates and species concentrations for the MZM and CFD simulations. This mechanism is chosen for several reasons. First, the conditions within RCM experiments HCCI engines are similar, and therefore the mechanism is valid for simulating RCM experiments. Second, the mechanism is relatively compact, containing only 33 species and 38 reactions, and is therefore feasible for CFD simulations. Last, the mechanism is still able, however, to simulate the low-temperature reactions necessary for producing multi-stage ignition and preliminary heat release.

\subsection{Computational Fluid Dynamics Simulations}

Data from CFD simulations of RCM experiments for the cases presented in the previous section are compared with data from MZM simulations in order to evaluate the predictive accuracy of the MZM. Converge CFD software is used to perform the simulations. The computational domain for the simulations is a 30-degree sector geometry representing the reaction chamber, gap and crevice of the RCCEM at Marquette University. Exact dimensions for the RCCEM are provided in Table 2.1. A sector size of 30-degrees was chosen in order to ensure an aspect ratio of near-unity for cells near the border of the mesh. It was found that smaller sector sizes resulted in many cells with high aspect ratio when using Converge's automatic cut-cell Cartesian grid generation technique, causing numerical dispersion errors. Converge generates a mesh by immersing the surface geometry of the simulation inside a block of cubic Cartesian 
cells, and then trimming around the edges. Cells along the edges that have a volume less than $30 \%$ of an interior neighbor cell are joined with that neighboring cell. [Richards, $\mathrm{K}$. J., Senecal, P. K. \& Pomraning, 2014]. An illustration of the computational domain is provided in Figure 4-1.

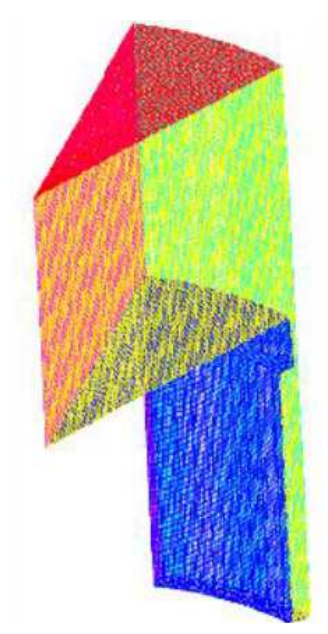

Figure 4-1: 30-degree sector computational domain utilized for CFD simulations [Neuman, 2015]

The base cell size used in the computational grid is $\mathrm{dx}=\mathrm{dy}=\mathrm{dz}=0.65 \mathrm{~mm}$. However, the advanced mesh refinement feature in Converge allows for grid refinement in areas where high fluctuation of certain parameters is observed based on sub-grid calculations. The sub-grid value of a scalar or vector variable is the difference between the value in the actual field and the value in the resolved field. Therefore, sub-grid values of a variable will be high in areas with a high spatial gradient. The equation for sub-grid values is an infinite series, therefore sub-grid values can only be estimated from finite terms. Details regarding how Converge estimates sub-grid values is provided in the theory manual [Richards, K. J., Senecal, P. K. \& Pomraning, 2014]. The simulations in this study utilize mesh refinement on the basis of temperature and velocity in order to achieve a finer grid at areas with a large temperature gradient (such as near the walls) and 
areas of high velocity gradient (such as inside the tapered gap). Specifically, the length of each dimension of a cell is reduced to $1 / 8^{\text {th }}$ of the original length if the difference between the sub-grid estimation of the temperature or velocity and the actual grid value is greater than 1. Embedding is removed if the sub-grid value is above 0.2. In order to maintain tractability of the simulation, a maximum limit of 750,000 cells was set.

Permanent refinement is also applied near the boundaries (cylinder and piston walls) in order to capture the temperature gradients in these areas caused by heat loss to the boundaries. The base grid size at the boundaries is $\mathrm{dx}=\mathrm{dy}=\mathrm{dz}=0.325 \mathrm{~mm}$, and this base grid size extends three layers into the interior of the mesh. It should be noted that adaptive mesh refinement applies to the permanently refined areas near the boundary in addition to interior cells. The overall grid parameters used for the CFD simulations were determined by simulating case 6 (n-heptane fuel, compression ratio of 11) using many different base grid sizes with different levels of both permanent and adaptive refinement. It was determined that the grid parameters described above created an adequately refined grid, and that further refinement produces less than $2 \%$ in ignition delay time.

Laminar flow conditions are used for the CFD simulations, as this approach has been shown to yield the best agreement with experimental results. In one study, a laminar and a turbulent law-of-the-wall model were used to simulate an RCEM experiment, and the results were compared to experimental data. The magnitude of pressure oscillations from acoustic waves yielded from pressure transducer in the experiment were of much lower magnitude than the turbulent predictions, and were of similar magnitude as the laminar predictions, indicating that the turbulent model overpredicts heat loss to the walls [S Scott Goldsborough \& Potokar, 2007]. In another study, 
turbulent models were shown to "smear out" the temperature and velocity profiles, as well as fail to predict the presence of vortices that were shown to exist in experiments [Mittal et al., 2008].

The transport equations are solved implicitly using the Pressure Implicit with Splitting of Operators (PISO) method. Converge uses a variable time step which is governed by the convective, sound speed, and diffusive Courant-Friedrichs-Lewy (CFL) numbers and the grid spacing. The maximum and minimum allowable time steps for the simulations were $10^{-4}$ seconds and $10^{-8}$ seconds, respectively. The SAGE detailed chemistry solver is used to simulate chemical evolution. A multi-zone model is utilized for the chemistry solver, which solves a single set of chemistry equations for all zones within a temperature bin of $1 \mathrm{~K}$, and an equivalence ratio bin of 0.025 . Without utilizing this multi-zone model, simulating chemistry within a refined mesh would simply be intractable.

A periodic boundary condition is applied to the tangential boundaries of the sector, while a constant temperature boundary condition is applied to the boundaries representing the cylinder and piston walls. The no-slip boundary condition is applied to the cylinder walls and the piston. Each CFD simulation begins with the specification of the initial temperature, pressure and species concentrations, which are uniform throughout the mesh. The mesh is then compressed according to the given piston trajectory that provides the velocity of the piston at a given time. Once the piston is compressed, the simulation continues until combustion occurs. 


\section{Chapter 5 Simulation Results}

\subsection{MZM Grid Study}

As discussed in Chapter 2, adequate grid refinement is required to accurately model temperature gradients and conduction in MZM simulation. Grid dependence is investigated in this study by examining maximum temperature and main reaction chamber pressure profiles for simulations of case 6 with varying numbers of zones. Figure 5-1 shows the maximum temperature profiles, while Figure 5-2 shows pressure. The figures are scaled to emphasize the ignition delay period, since this is where discrepancies between simulations with different numbers of zones are found. Temperature and pressure profiles prior to approximately 1 millisecond before top dead center are nearly identical, regardless of the number of zones used in the simulations.

As can be seen from the figures, a greater number of zones results in a lower temperature and pressure at top dead center, as well as during the ignition delay period. This is due to the distance of the thermal gradients between each zone being larger for a less refined grid, resulting in less conduction, and therefore less heat loss from the main reaction chamber. In Figure 5-1, the maximum main reaction chamber temperature at top dead center is $\sim 655 \mathrm{~K}$ for a simulation that uses 5 zones, while for 60 zones, the temperature is $\sim 654 \mathrm{~K}$. However, further refinement beyond 15 zones yields insignificant change in the results. The results for 40 and 60 zones are indistinguishable.

From Figure 5-2, it is seen that the main reaction chamber pressure at top dead center is practically identical for simulations using 15 and 60 zones. Figure 5-2 shows a difference in main reaction chamber pressure at top dead center of less than 0.05 bar 
between 15 and 60 zones. The temperature and pressure profiles for 15 and 60 zones begin to diverge as the simulations move further into the post-compression period, but the divergence is very limited, resulting in an ignition delay difference of less than 0.1 milliseconds, or $0.5 \%$. In order to ensure that the MZM grid is sufficiently refined without resulting in overly computationally expensive simulations, each simulation in this study is run with 20 zones. The thickness of the outermost zones for these simulations is $0.18 \mathrm{~mm}$. For the given number of zones and outermost zone thickness, and bore value of 2 inches, the value of the growth factor, $\alpha$, for Equation 3.1 is 1.1768.

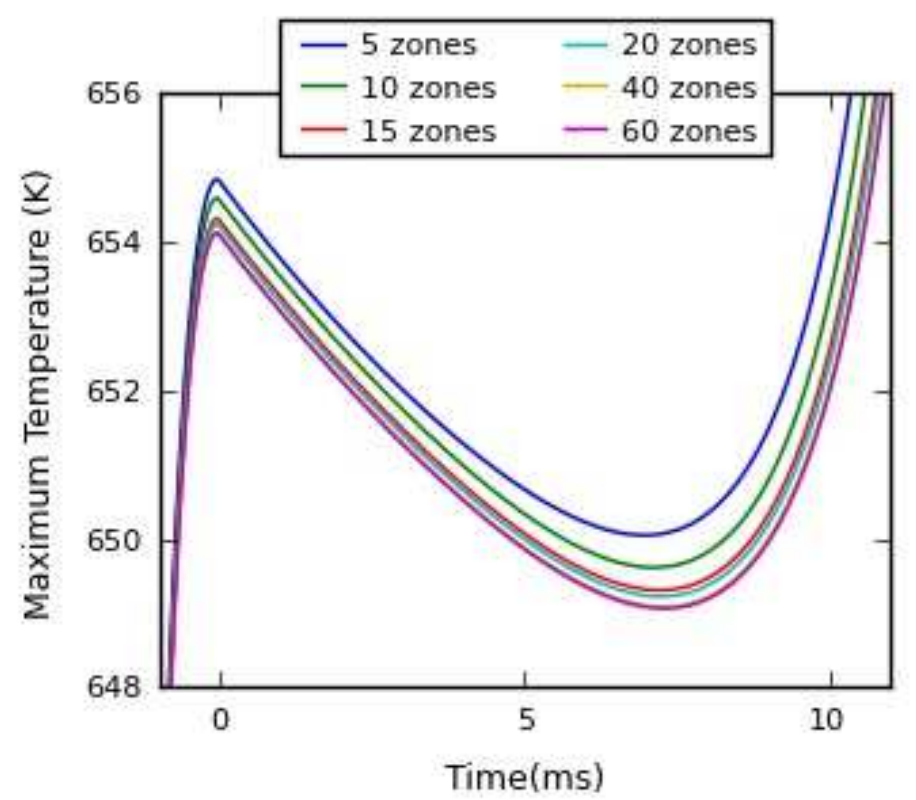

Figure 5-1: MZM maximum temperature versus time for case 6 for varying numbers of zones. 


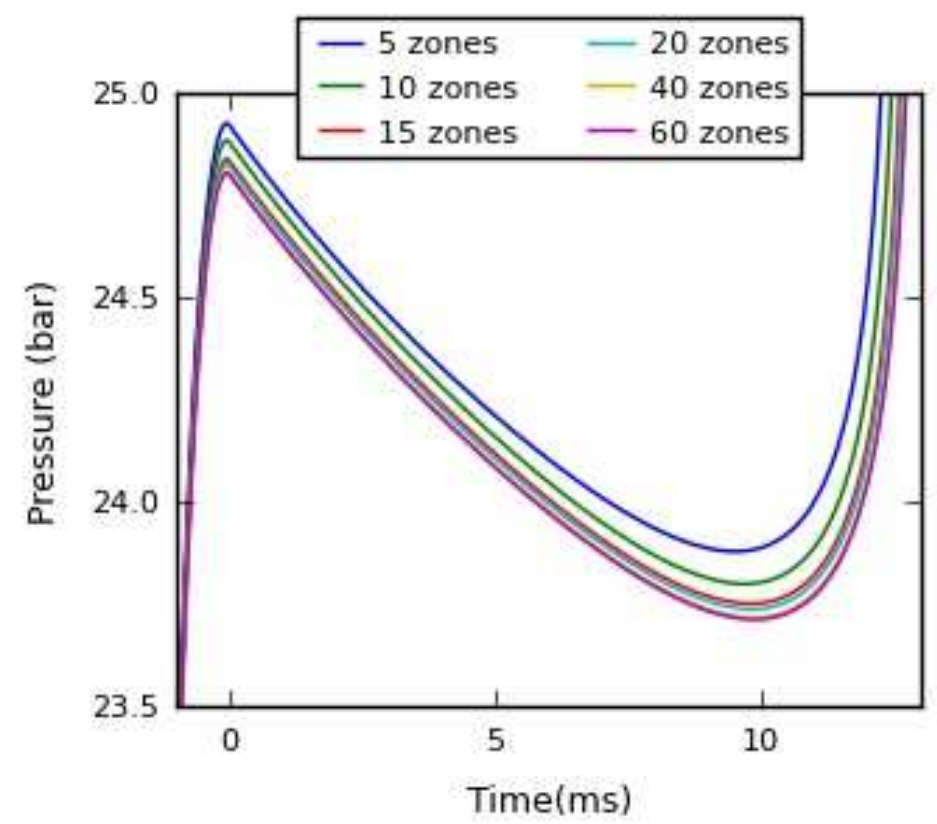

Figure 5-2: MZM pressure versus time for case 6 for varying numbers of zones.

\subsection{Validation of MZM Pressure Prediction}

Pressure in the main reaction chamber predicted by the MZM and CFD are compared for each test case. Accurate main reaction chamber pressure predictions are indicative of the ability of the MZM to model physical processes in the main reaction chamber, especially conduction and mass flow to the crevice, both of which greatly affect pressure. In the MZM simulations, the energy loss from the main reaction chamber due to mass flow to the crevice is more significant than conductive heat loss during the last 5 ms of compression. The peak enthalpy loss rate is at least 3 times greater than the peak conductive heat loss rate during this time period. However, during the delay period, the conductive heat loss rate is about $20 \%-40 \%$ greater than the enthalpy loss rate. It is worth noting that mass flow to the crevice reduces the total mass in addition to the internal energy in the main reaction chamber, compounding its effect on pressure. 
Conductive losses only affects the internal energy. Pressure in the main reaction chamber is strongly linked to average temperature and total mass, both of which significantly affect species concentrations, which is proposed as a more rigorous parameter for validating kinetic models.

Figures 5-3 illustrates the main reaction chamber pressure with respect to time for each $n$-heptane/air case. Excellent agreement between the MZM and CFD predictions during compression can be observed. The MZM main reaction chamber pressures continue to agree very well with the CFD prediction for the post compression period, although slight deviations are present. These deviations grow with respect to time, causing a greater difference in ignition time between the two models for the compression ratio of 9 case, which has a longer ignition delay. The pressure profiles for the $n$ heptane/air mixture generated by CFD and MZM simulations are nearly identical for compression ratios of 10 and 11, which yield ignition delays of approximately $26 \mathrm{~ms}$ and $13 \mathrm{~ms}$, respectively. However, for a compression ratio of 9 , the ignition delay is approximately $60 \mathrm{~ms}$ and $63 \mathrm{~ms}$ for the MZM and CFD, respectively.

This same general trend can be observed for the simulations involving iso-octane and the iso-octane/ $n$-heptane mixture, as shown in Figures 5-4 and 5-5. These simulations also demonstrate the ability of the MZM to simulate multi-stage ignition, although the two stage ignition behavior is much more subtle for the $i s o$-octane $/ n$-heptane mixture cases. For the iso-octane cases, as shown in Figure 5-4, the MZM pressure trace is nearly indiscernible from that of the CFD simulation for a compression ratio of 13 . Pressure of the MZM for the other two compression ratios is slightly under predicted due to these cases having longer ignition delay times. However, the agreement for the two 
cases is still very good, with the difference in ignition delay being less than $3.5 \%$ for both first stage and second stage ignition for the compression ratio of 12 case, and less than $4.5 \%$ for the compression ratio of 11 case.

The MZM also slightly under predicts pressure for the $i s o$-octane $/ n$-heptane blend case where compression ratio is 9, as seen from Figure 5-5. However, the CFD and MZM pressure traces match exceptionally well for the other two compression ratios. Overall, the MZM accurately captures the effects of conduction, mass flow to the crevice, and chemical reaction in order to predict pressure in the main reaction chamber. The MZM directly accounts for the effects of chemical ignition throughout the entire main reaction chamber, unlike the original model, making it more suitable for cases with multistage ignition.

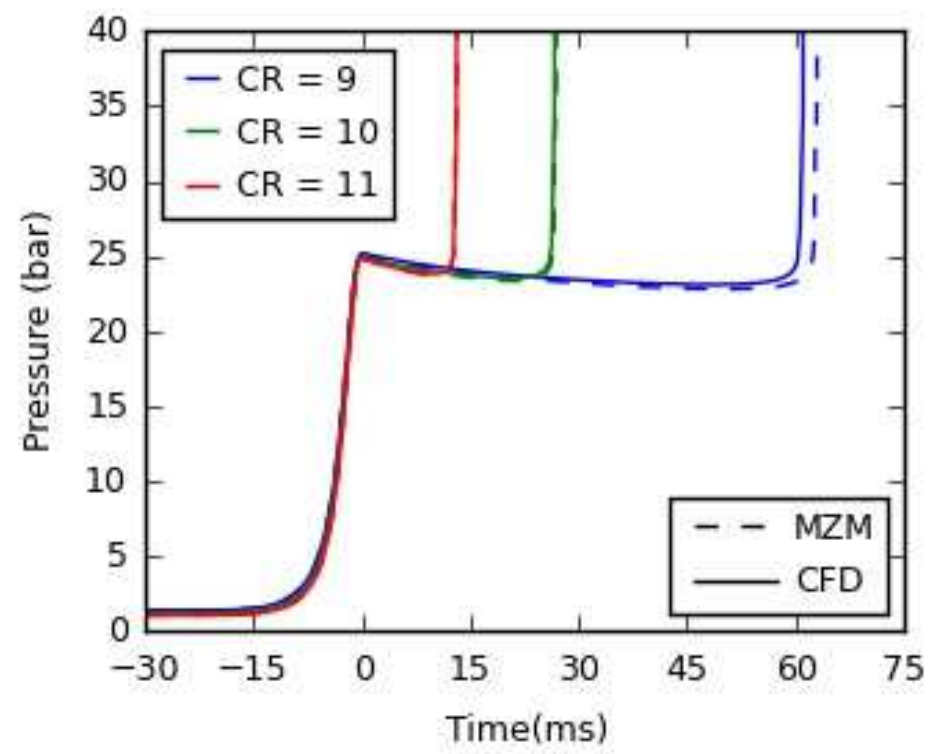

Figure 5-3: Predicted main reaction chamber pressures for MZM and CFD RCM simulations with a stoichiometric mixture of $n$-heptane and air at compression ratios of 9 , 10 and 11 . 


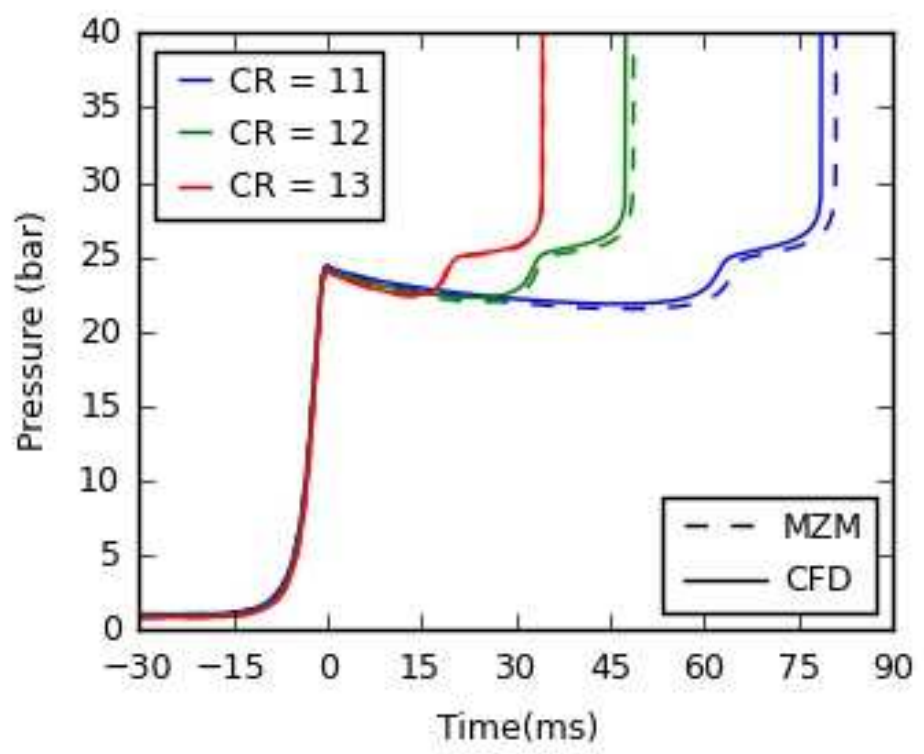

Figure 5-4: Predicted main reaction chamber pressures for MZM and CFD RCM simulations with a stoichiometric mixture of iso-octane and air at compression ratios of 11,12 , and 13.

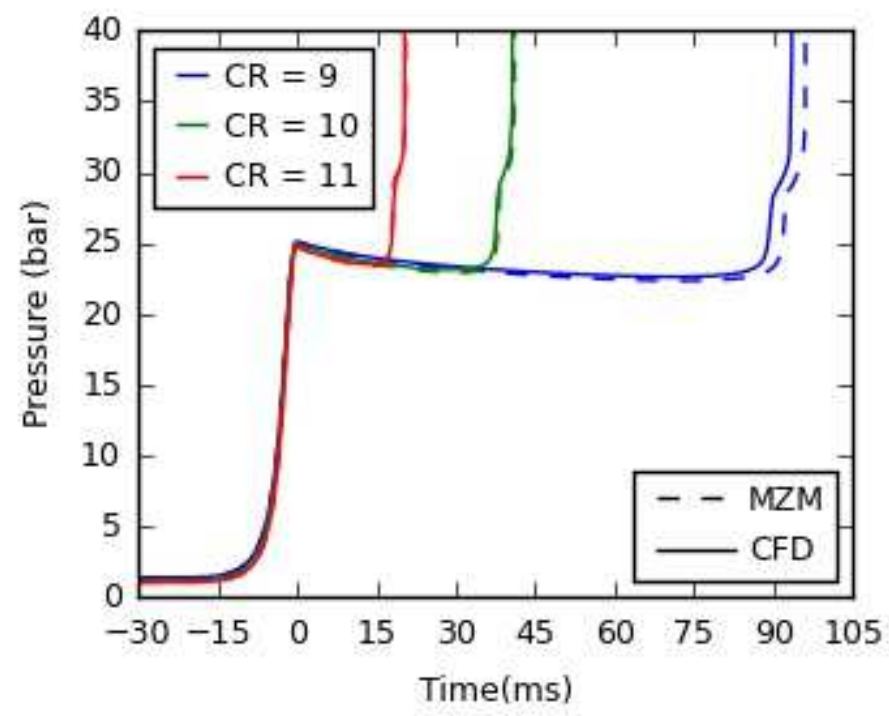

Figure 5-5: Predicted main reaction chamber pressures for MZM and CFD RCM simulations with a stoichiometric mixture of fuel and air at compression ratios of 9, 10, and 11 . The fuel is a mixture of $50 \%$ by volume iso-octane and $50 \%$ by volume $n$ heptane. 


\subsection{Validation of MZM Maximum Temperature Prediction}

In many low temperature RCM experiments, especially ones with temperatures that lie outside the negative temperature coefficient (NTC) region, a significant amount of the chemical reaction occurs in the core region, where the high temperatures exists. Therefore, accurate maximum temperature predictions are imperative for chemical species calculations. Maximum temperature also generally governs ignition delay time, the typical criterion by which kinetic models are currently validated. As stated above, pressure data is telling of the average conditions within the main reaction chamber.

While average and maximum temperature are certainly related, the accurate prediction of one does not necessarily guarantee the accurate prediction of the other, since average conditions are affected by both the core and boundary layer. Therefore, maximum temperature of the MZM must be validated as well.

Maximum main reaction chamber temperature profiles for MZM and CFD simulations for the $n$-heptane cases are shown in Figures 5-6. The MZM predicts a maximum temperature profile that closely matches the maximum temperature profile of the CFD simulations. Again, a slight deviation between the MZM and CFD simulations is present in the delay period, where this deviation becomes more pronounced as the simulation moves further in time, causing the MZM to slightly under predict maximum temperature for simulations with longer ignition delay times. For each compression ratio in Figure 5-6, the maximum temperature predicted by the MZM at top dead center is within a $0.35 \%$ difference with respect to the maximum temperature predicted by the respective CFD simulations. The difference remains low until the beginning of ignition for compression ratios 10 and 11 , resulting in very similar ignition delay times between 
MZM and CFD. However, for a compression ratio of 9, the difference in maximum temperature between the MZM and CFD increases during post compression, causing an ignition delay difference of about $3 \mathrm{~ms}(\sim 5 \%)$.

The iso-octane cases display a similar trend, as shown in Figure 5-7. Percent differences between MZM and CFD remain below 0.5\% during the post-compression period before temperature increase from ignition occurs for compression ratios of 12 and 13. This leads to close ignition times between MZM and CFD for both first and second stage ignition. For the compression ratio of 11 case, the percent difference before temperature increase from ignition occurs is about $0.65 \%$. The difference in first stage ignition time is about $6 \%$. Again, the trend for the $n$-heptane/iso-octane cases are very similar, with the greatest ignition delay difference between the MZM and CFD being for the case with the smallest compression ratio $(\mathrm{CR}=9)$.

One explanation of the greater discrepancy seen for both maximum main reaction chamber temperature and pressure between MZM and CFD simulations with longer ignition delay times could be the error associated with the tapered gap and crevice models. The conditions within the crevice heavily influence the main reaction chamber conditions, since pressure difference between the main chamber and crevice drives mass flow between the two regions. As the simulation progresses, the effect of these errors compound. The fact that the MZM is under predicting main reaction chamber temperature and pressure could mean that the main reaction chamber is losing too much enthalpy to the crevice via mass flow during the delay period. This seems reasonable, considering the Nusselt number of the crevice remains high in the latter parts of the simulation, even when crevice velocity is low, due to the first term in the Nusselt number 
equation, which is independent of crevice velocity (see Eq. 1). Therefore, perhaps the MZM is over predicting the overall heat loss in the crevice during the delay period.

Nevertheless, the MZM predicts maximum main reaction chamber temperature very well with respect to CFD simulations, with the difference in ignition delay being about $6 \%$ in the worst case.

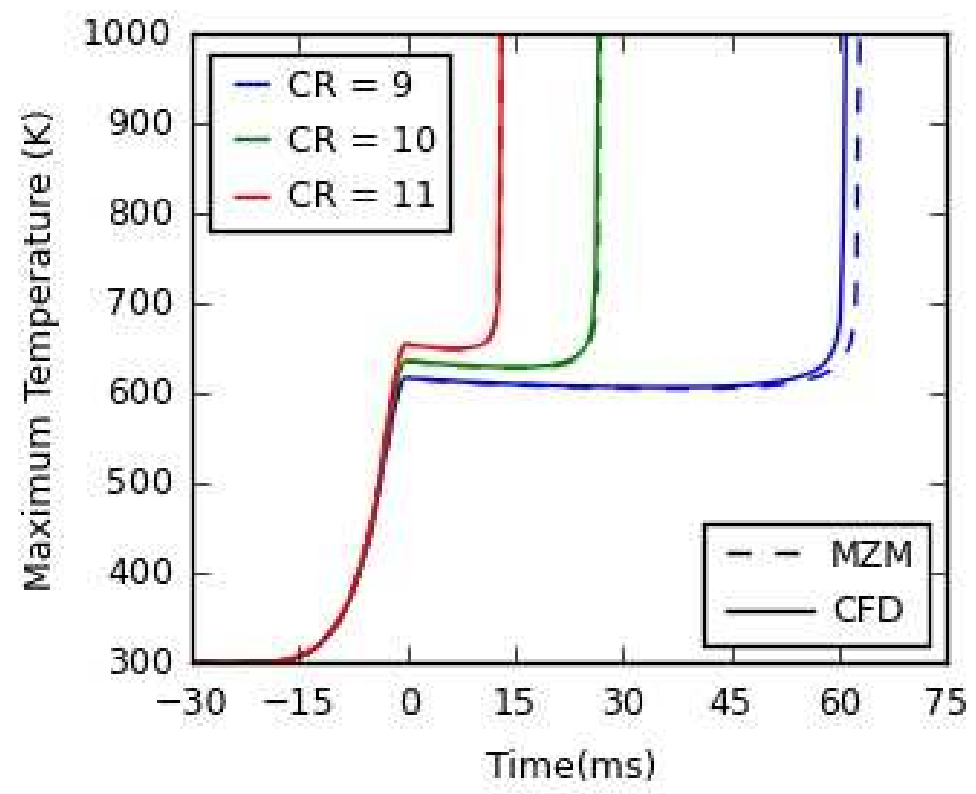

Figure 5-6: Maximum main reaction chamber temperature for RCM simulations with a stoichiometric mixture of $n$-heptane and air at compression ratios of 9, 10 and 11 . 


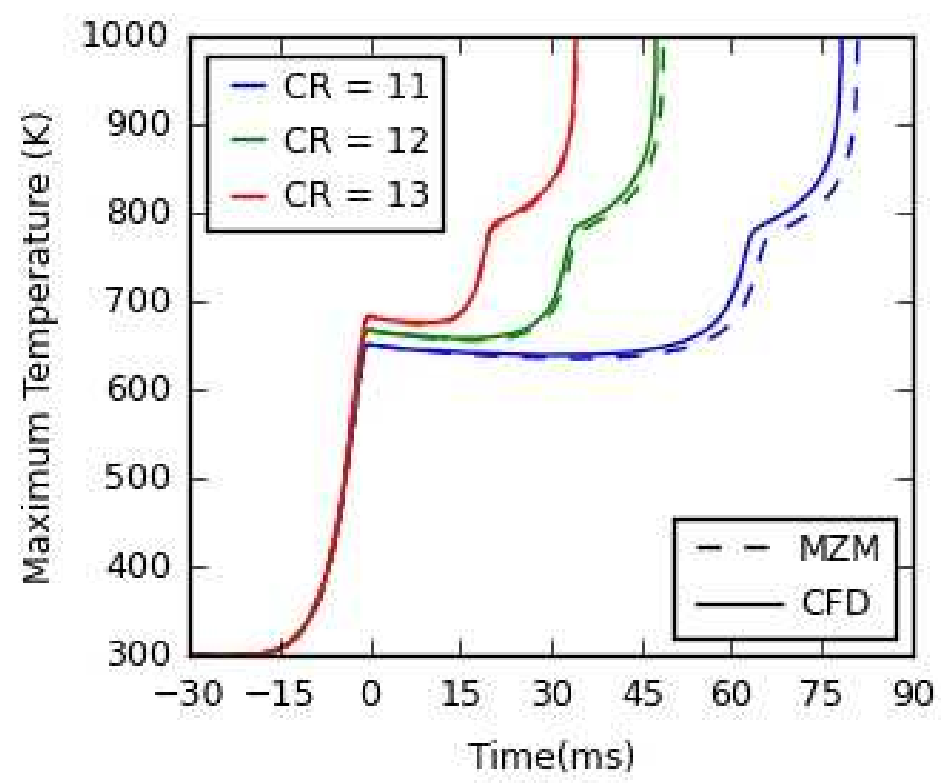

Figure 5-7: Maximum main reaction chamber temperature for RCM simulations with a stoichiometric mixture of iso-octane and air at compression ratios of 11,12 , and 13 .

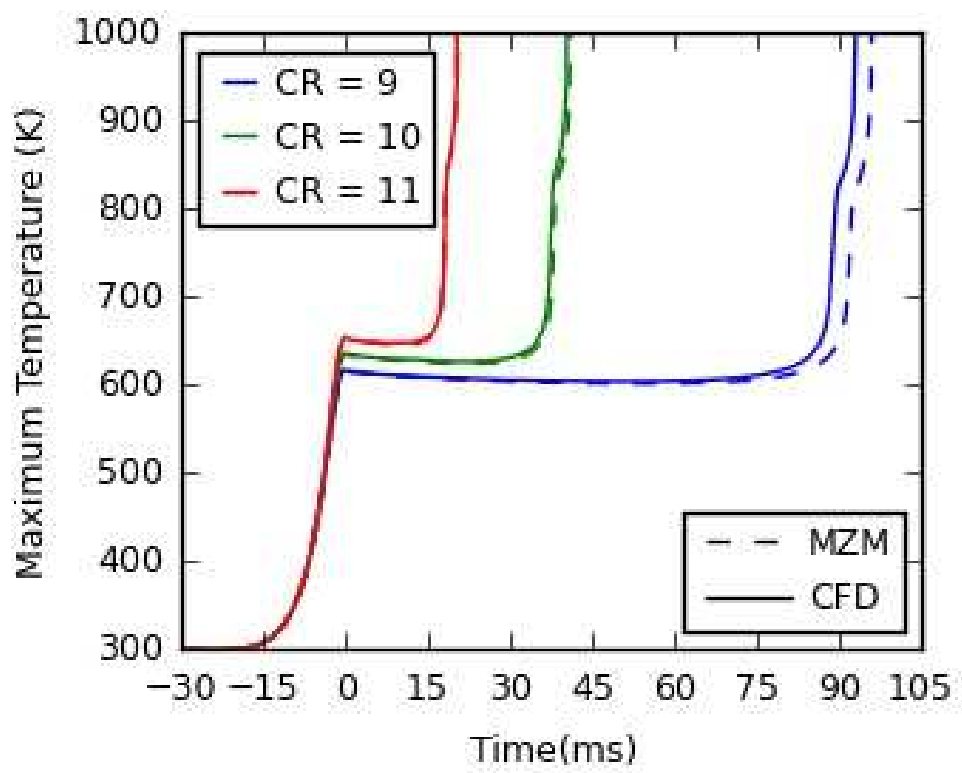

Figure 5-8: Maximum main reaction chamber temperature for RCM simulations with a stoichiometric mixture of fuel and air at compression ratios of 9,10 , and 11 . The fuel is a mixture of $50 \%$ by volume iso-octane, and $50 \%$ by volume $n$-heptane. 


\subsection{Temperature Distribution Comparison between MZM and CFD}

Chemical reaction rates vary considerably with temperature. Therefore, in order to predict species concentrations, it is important for the MZM to be able to accurately model the temperature distribution in RCM experiments. Figure 5-9 illustrates the temperature distribution at various times for the iso-octane, compression ratio of 11 case for the MZM and CFD. This case is shown since it produced the least agreement in ignition delay times between MZM and CFD for the iso-octane cases.

The figure shows the fraction of volume a particular MZM zone at a single temperature occupies in the main reaction chamber. For the CFD results, the figure shows the volume fraction occupied by specific temperature ranges. The maximum and minimum of each CFD temperature range is set as the median temperature between neighboring MZM zones, aside from the highest temperature range, whose maximum is set as the maximum CFD temperature at that time. Setting the maximum and minimum of each CFD temperature range in such a way allows for a good comparison between MZM and CFD temperature distributions. It should also be noted that, due to many zones being at or close to the maximum temperature, for clarity, volume fractions of MZM zones with a temperature within $2 \%$ of the maximum temperature are combined. The temperature of this combined zone is presented as the average temperature of the original zones.

As seen from the figure, the MZM predicts the fraction of volume at or near the maximum temperature reasonably well at each time. However, at $0 \mathrm{~ms}$, the point representing the maximum temperature MZM zone is located at the upper right corner of the rightmost CFD bin. This means that the volume occupied by the temperature range 
represented by the rightmost bin for the CFD simulation is nearly equal to the volume occupied by the maximum temperature for the MZM simulation, suggesting that more volume could be at or very near to the maximum temperature for MZM simulations. As the simulation progresses, the location of the point representing the maximum temperature MZM zone shifts left relative to the right boundary of the CFD bin, as the CFD predicts higher maximum temperatures.

Aside from the maximum temperature bin, the MZM appears to generally predict a slightly lower occupied volume than CFD for higher temperatures, and a higher occupied volume than CFD for lower temperatures. For example, at $0 \mathrm{~ms}$, the volume percentages of the second zone/bin from the right for the MZM and CFD are $2.5 \%$ and $3.7 \%$, respectively. The volume percentages of the coolest zone/bin for the MZM and CFD are $1.5 \%$ and $0.05 \%$, respectively. Agreement improves as the simulation progresses. At $35 \mathrm{~ms}$, the volume percentages of the second zone/bin from the right for the MZM and CFD become $4.2 \%$ and $4.9 \%$, respectively. At 50 and $65 \mathrm{~ms}$, the maximum temperature bin is no longer near the upper right corners of the CFD bin. This is because these two times occur during first and second stage ignition, respectively, and the CFD has progressed further in ignition at these times and therefore has a higher maximum temperature. The overall trend of higher temperatures occupying greater volume fractions exhibited by the CFD data is reflected in the MZM data. 

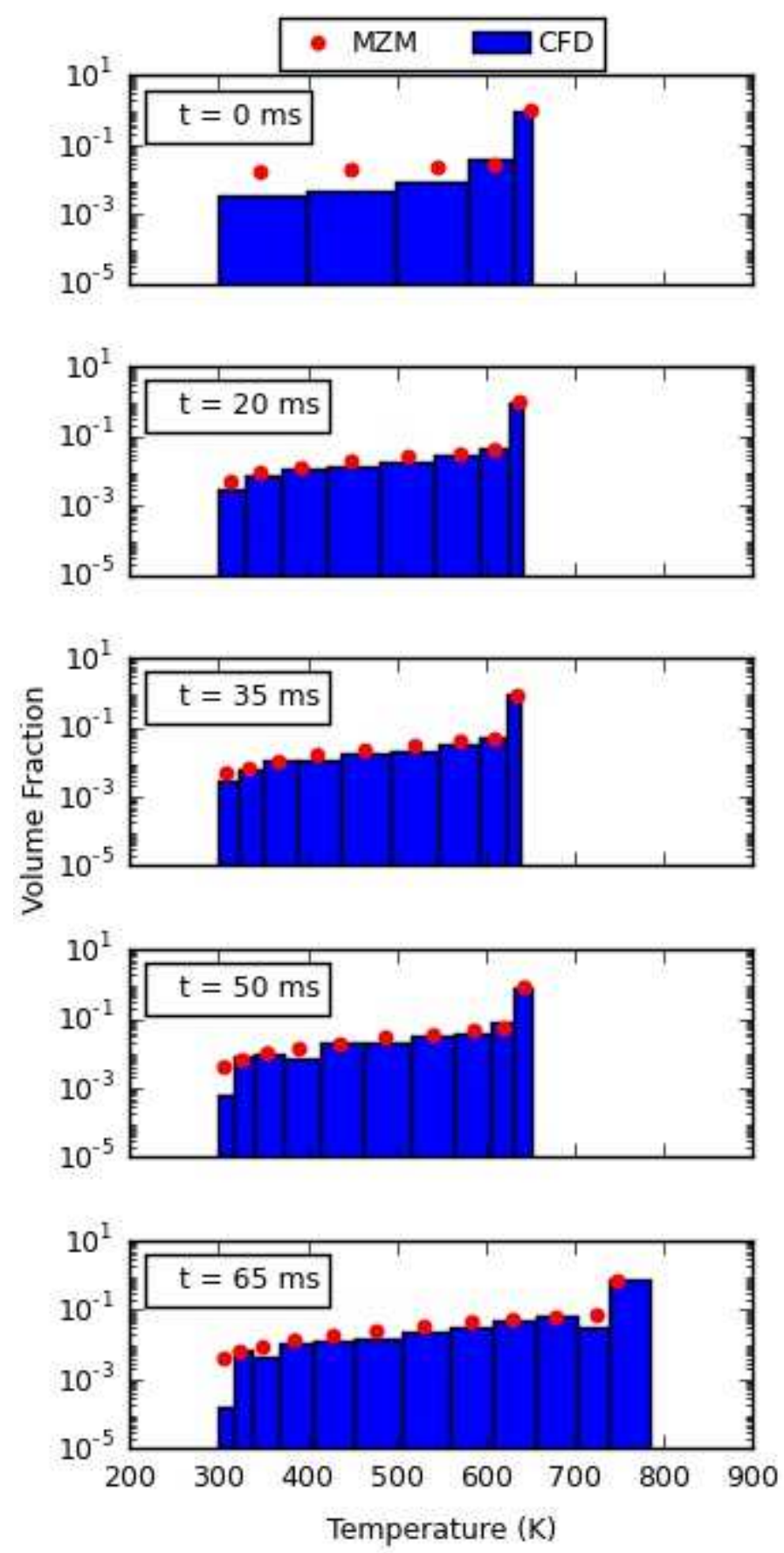

Figure 5-9: Fraction of main reaction chamber volume occupied by single temperatures for the MZM, and temperature ranges for CFD, for the iso-octane, compression ratio of 11 case. The MZM volume fractions for each individual zone are represented by the red points, while CFD volume fractions are represented by the blue bars. 


\subsection{Validation of MZM Chemical Species Prediction}

The intended application of the MZM is to simulate RCM quenching experiments. Therefore, the MZM should be able to accurately predict chemical species. Presented below are comparisons of speciation data for MZM, CFD, and HRM simulations for each case. Specifically, predicted average mole fractions of fuel $\left(\mathrm{C}_{7} \mathrm{H}_{16}\right.$ and/or $\left.\mathrm{C}_{8} \mathrm{H}_{18}\right)$, carbon monoxide $(\mathrm{CO})$, formaldehyde $\left(\mathrm{CH}_{2} \mathrm{O}\right)$, and hydroxyl $(\mathrm{OH})$ for the entire $\mathrm{RCM}$ are compared for each case. For the HRM simulations, the volume profile is calculated using the isentropic relationship between pressure and volume change. The pressure profile is generated from an MZM simulation that is void of chemical reaction, just as in the model by Goldsborough et al [S. Scott Goldsborough et al., 2012]. It is important to note differences in ignition delay between simulations when comparing them in terms of species concentrations, since ignition delay is indicative of the rate of chemical progression. Ignition delay data is for the MZM, HRM and CFD for each case are given in Table 5-1. 


\begin{tabular}{|c|c|c|c|}
\hline \multirow{2}{*}{ Case } & \multicolumn{3}{|c|}{ Ignition Delay Time (ms) } \\
\cline { 2 - 4 } & MZM & HRM & CFD \\
\hline 1 & 81.5 & 69.0 & 78.0 \\
\hline 2 & 49.0 & 43.5 & 47.5 \\
\hline 3 & 34.5 & 30.0 & 34.5 \\
\hline 4 & 63.0 & 58.0 & 60.0 \\
\hline 5 & 26.0 & 24.5 & 26.0 \\
\hline 6 & 13.0 & 13.0 & 13.0 \\
\hline 7 & 96.5 & 87.0 & 93.0 \\
\hline 8 & 41.0 & 37.0 & 40.5 \\
\hline 9 & 20.5 & 18.0 & 20.5 \\
\hline
\end{tabular}

Table 5-1: Ignition delay times for each case for MZM, HRM, and CFD simulations. For cases with multiple stages of ignition, ignition delay time is taken as the maximum change in pressure with respect time for the final ignition event.

Figure 5-10 illustrates the comparison of mole fractions of the aforementioned species for the $\mathrm{n}$-heptane cases. It is seen that the MZM $n$-heptane mole fraction profile follows closely with the predictions by CFD simulations for $n$-heptane cases, even during the latter parts of combustion. This is especially true for compression ratios of 10 and 11 . However, noticeable discrepancy exists between MZM and CFD $n$-heptane mole fraction predictions near combustion for a compression ratio of 9, although the overall trajectories are similar. This discrepancy is due to the differences in ignition delay between the two models. Ignition time is dictated by the maximum temperature in the main reaction chamber. As discussed above, the deviation in maximum temperature between MZM and CFD simulations increase as simulation time increases due to the errors associated with the simplifying assumptions of the MZM.

Figure 5-10 also shows predictions of carbon monoxide, formaldehyde and hydroxyl mole fractions for the $n$-heptane cases. The MZM accurately predicts the 
magnitude of each of these intermediate species during the ignition delay period, with the average percent difference in mole fraction prediction between MZM and CFD considering all compression ratios being about $10 \%$. Better agreement between the MZM and CFD exists for lower compression ratios. It should be noted that from here on, percent differences in mole fractions between the MZM/HRM and CFD simulations are calculated as $\frac{X_{M Z M / H R M}-X_{C F D}}{X_{C F D}}$.

For the HRM, significant disagreement in $n$-heptane mole fraction with both CFD and MZM exists for compression ratios 9 and 10. This is also due to differences in ignition delay. The early ignition time predicted by the HRM is due to the HRM neglecting enhanced heat transfer that's due to chemical heat release. The average percent difference between the HRM and CFD for each intermediate species before ignition is about $80 \%$, which is poorer than the agreement between the MZM and CFD. Some of this discrepancy be can explained by the differences in ignition time between the HRM and CFD predictions, since these two models are at different points in the reaction path at a given time.

However, there is still some difference in intermediate species mole fractions for the compression ratio of 11 case, even though ignition time predicted by the HRM and CFD agree very well. The agreement in ignition timing between the HRM, MZM and CFD is indicative from the similar timing of the spike in intermediate species mole fractions for each simulation type. However, despite the agreement in ignition delay, the average percent difference between the HRM and CFD for the compression ratio of 11 case for each intermediate species is about $50 \%$, compared to $10 \%$ between the MZM and CFD. The discrepancy in this case is attributed to the fact that the HRM is predicting 
core conditions, which do not reflect average conditions throughout the entire RCM, making the HRM unsuitable for predicting speciation data in an RCM quenching experiment. 


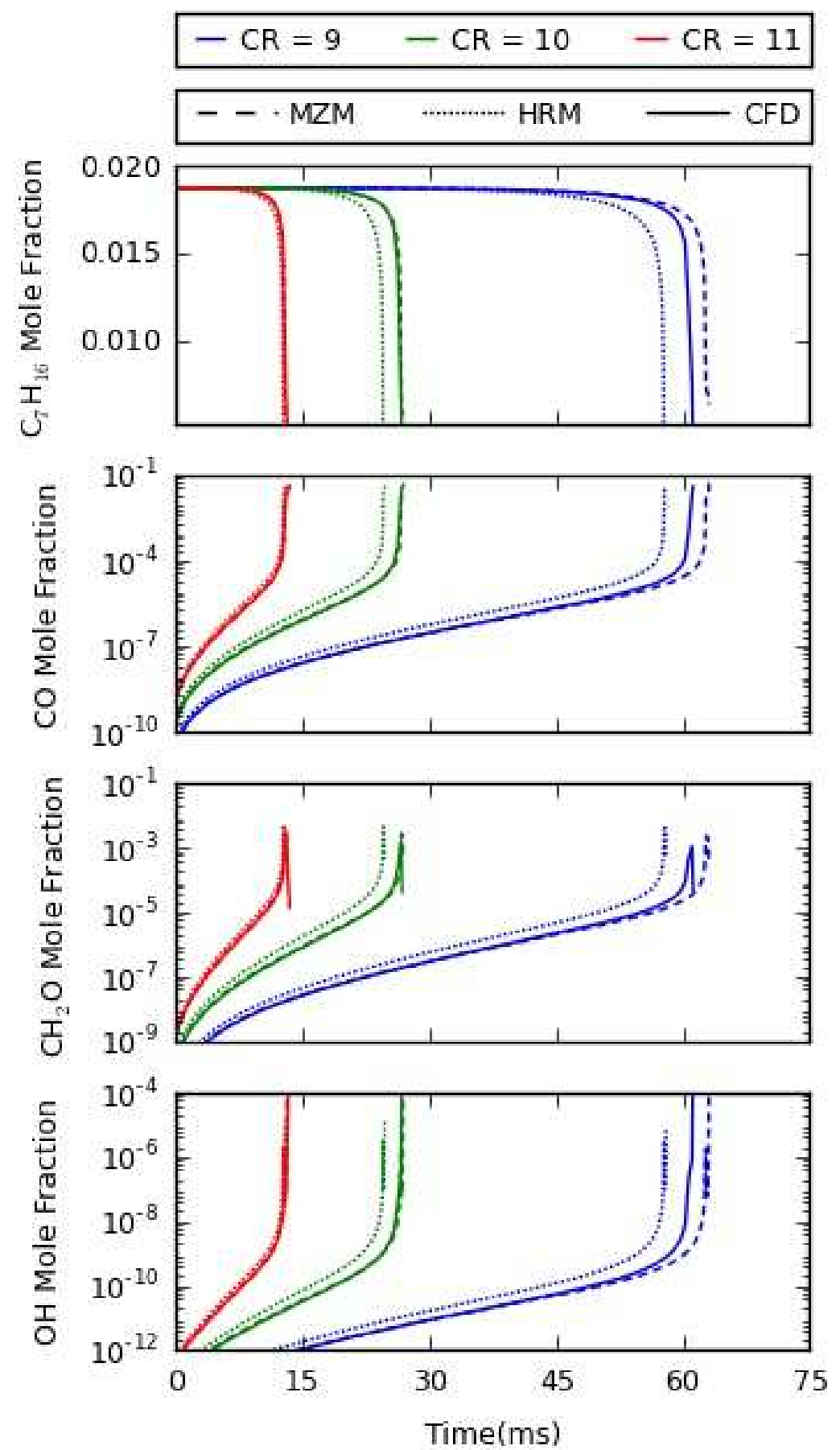

Figure 5-10: MZM, HRM, and CFD predictions of mole fractions of $n$-heptane $\left(\mathrm{C}_{7} \mathrm{H}_{16}\right)$, oxygen $\left(\mathrm{O}_{2}\right)$, formaldehyde $\left(\mathrm{CH}_{2} \mathrm{O}\right)$, and hydroxyl $(\mathrm{OH})$ radical for stoichiometric $n$ heptane/air cases.

Predictions of chemical species for iso-octane combustion are shown in Figure 5-

11. The MZM mole fraction profile for iso-octane agrees well with the CFD simulations 
throughout the entire post-compression period. Slight discrepancies in fuel consumed during first stage ignition, as well as the timing of second stage ignition are apparent in the iso-octane mole fraction profiles. Better agreement in the timing of second stage ignition, as noted by the second initiation of rapid fuel consumption, is seen for simulations with larger compression ratios which have shorter ignition delay times. Differences in ignition time also cause discrepancies in magnitudes of intermediate species mole fractions near first and second stage ignition for the iso-octane, compression ratio of 11 case. This is also true for second stage ignition in the compression ratio of 10 case. The compression ratio of 9 case has excellent agreement throughout the entire simulation. Overall, the magnitude and trajectory of intermediate species mole fraction profiles predicted by the MZM are consistent with CFD results for every case.

The average percent difference for each intermediate species between MZM and CFD predictions during pre and post first stage ignition is about 15\%. A worthwhile comparison of radical species mole fractions during first and second stage ignition is difficult for some cases, due to slight differences in ignition time. Similar to the $n$ heptane cases, better agreement between MZM and CFD radical species corresponds with lower compression ratios. Again, the HRM does a poor job predicting intermediate species mole fractions compared to the MZM, with the average percent difference between HRM and CFD predictions for each intermediate species being about $80 \%$ during pre and post first-stage ignition. Also, the HRM predicts a much greater magnitude of iso-octane mole fraction reduction than the MZM and CFD during first stage ignition. This is because the HRM simulates core conditions only, where much greater fuel consumption per unit mole occurs relative the rest of the RCM reaction 
chamber. As a reference point, it is noted that the percent errors of the MZM speciation predictions are well within the magnitude of errors in ignition delay time predictions that arise by using the HRM to simulate RCM experiments with two-stage ignition fuels [Mittal et al., 2010].

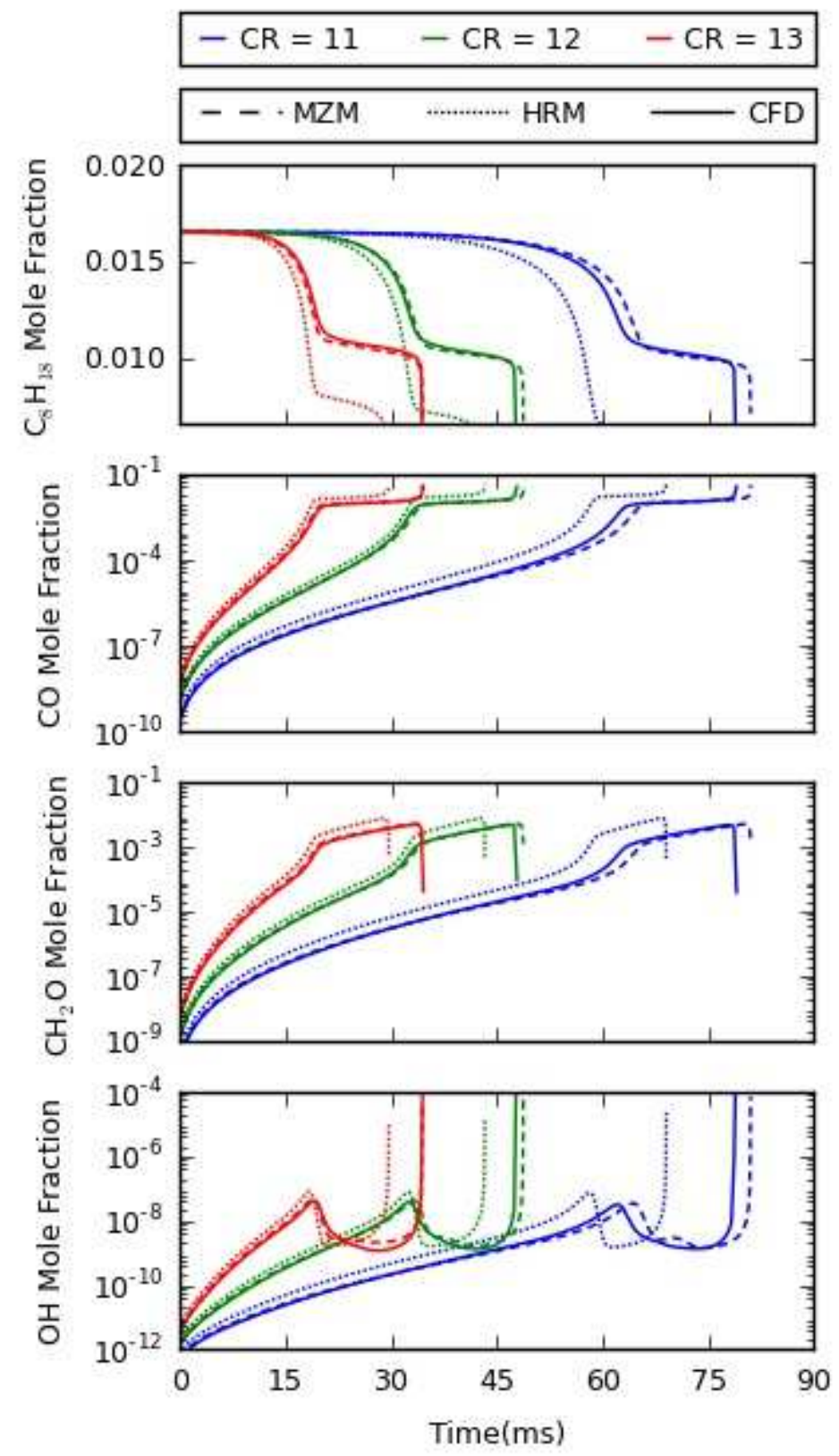

Figure 5-11: MZM, HRM, and CFD predictions of mole fractions of iso-octane $\left(\mathrm{C}_{8} \mathrm{H}_{18}\right)$, oxygen $\left(\mathrm{O}_{2}\right)$, formaldehyde $\left(\mathrm{CH}_{2} \mathrm{O}\right)$, and hydroxyl $(\mathrm{OH})$ radical for stoichiometric isooctane/air cases. 
The trends for $n$-heptane/iso-octane fuel blend cases are very similar to that of the iso-octane cases in regards to speciation data, as shown in Figure 5-12. The agreement between MZM and CFD mole fractions is good for each species. Again, as in the isooctane cases, differences in second stage ignition timing cause an offset between the MZM and CFD fuel mole fraction profiles for the case with the largest ignition delay time, which is the compression ratio of 9 case. However, the timing of second stage ignition between the MZM and CFD agrees well for the cases with compression ratios of 10 and 11. This is indicative from the overlay of the MZM and CFD iso-octane profiles during the second period of rapid iso-octane decomposition. The MZM does not predict a second stage of rapid $n$-heptane decomposition as the CFD simulation does, although the transition from rapid to gradual decomposition in the MZM $n$-heptane profile is apparent and the profiles for the two simulation types agree very well up until the initiation of the second stage rapid $n$-heptane decomposition predicted by CFD. Overall, the fuel mole fraction profiles predicted by the MZM mimic those predicted by the CFD for each case.

Figure 5-12 also shows that the intermediate species profiles predicted by the MZM and CFD agree well throughout the entire simulation for compression ratios 10 and 11. Good agreement in intermediate species profiles is also seen for the compression ratio of 9 case, up until first stage combustion, where differences in ignition delay cause the profiles to be skewed relative to one another. As in the cases for the other two fuels, lower overall percent differences in radical species mole fractions between MZM and CFD predictions exist for lower compression ratios for the fuel-blend cases. The average of these percent differences is about $15 \%$ up until first stage ignition for each radical 
species. Again, the agreement in intermediate species mole fractions between HRM and CFD is inferior, with the average percent difference being about $90 \%$. The HRM also predicts only one stage of rapid $n$-heptane decomposition which continues until the end of the simulation, unlike the MZM and CFD. For every case, the HRM over predicts radical species mole fractions compared to MZM and CFD simulations. This is due to the HRM predicting greater reaction progress, as well as the fact that the HRM represents the core gas in an RCM experiment, which has different conditions than the rest of the RCM domain. 


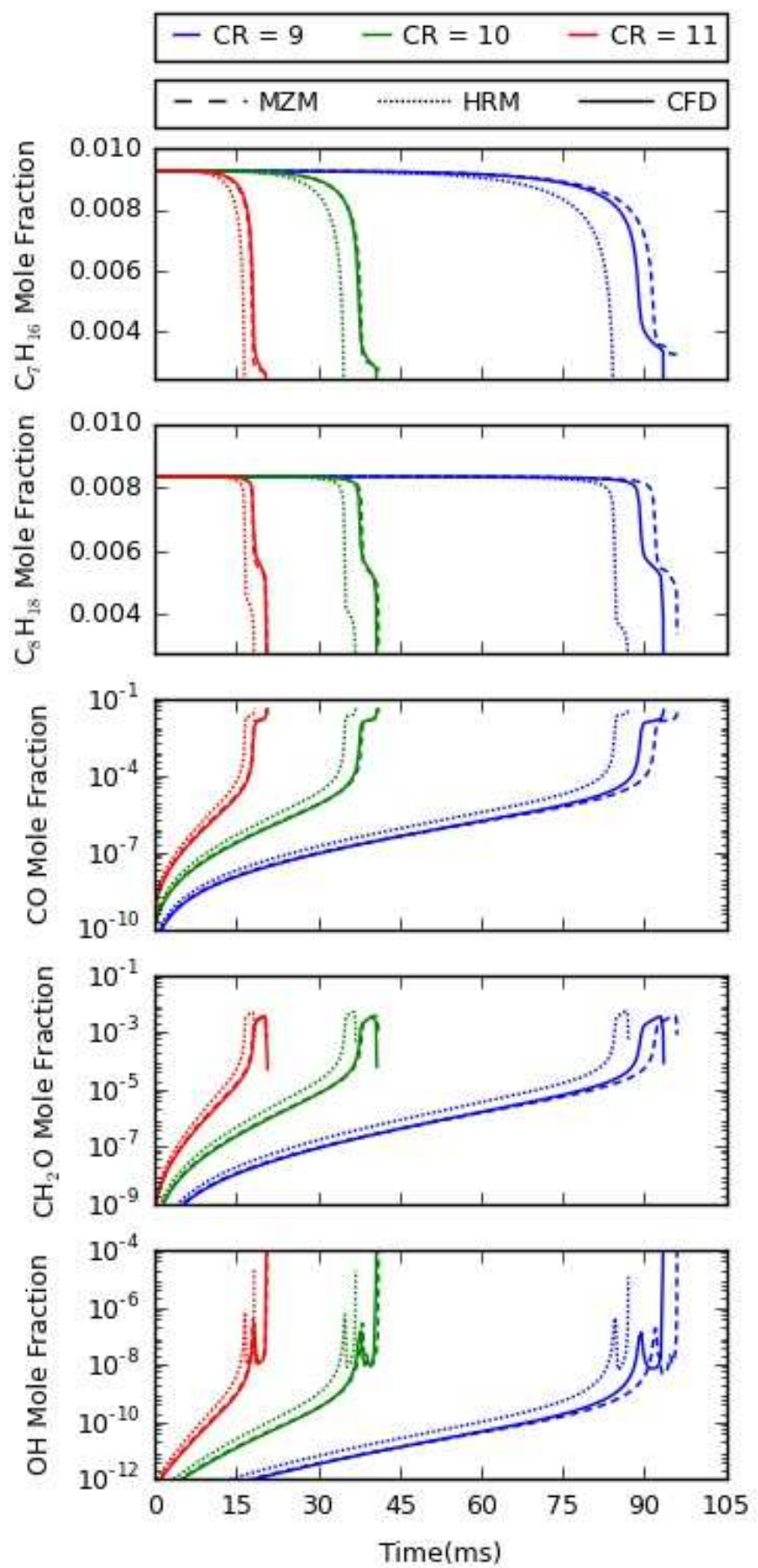

Figure 5-12: MZM, HRM, and CFD predictions of mole fractions of $n$-heptane $\left(\mathrm{C}_{7} \mathrm{H}_{16}\right)$, iso-octane $\left(\mathrm{C}_{8} \mathrm{H}_{18}\right)$, oxygen $\left(\mathrm{O}_{2}\right)$, formaldehyde $\left(\mathrm{CH}_{2} \mathrm{O}\right)$, and hydroxyl $(\mathrm{OH})$ radical for cases with a stoichiometric mixture of a fuel blend and air. The fuel blend consists of $50 \%$ by volume $n$-heptane, and $50 \%$ by volume iso-octane. 


\section{Chapter $6 \quad$ Summary \& Future Work}

\subsection{Summary}

A physics-based MZM for predicting chemical speciation data, in addition to temporal temperature and pressure profiles for RCM experiments is presented in this thesis. Species concentrations are a more rigorous parameter for comparing RCM simulation with experiment than ignition delay alone, and are therefore better for evaluating the accuracy of a chemical kinetic mechanism than temperature and pressure data. While details regarding reaction pathways and elementary reactions can only be inferred from temperature and pressure data, they can be directly obtained from speciation data. Prior reduced-order models utilize an HRM, which simulates the conditions inside the core region of the reaction chamber of an RCM. Therefore, these approaches are unsuitable for predicting speciation data for RCM quenching experiments. Also, the effective volume profiles utilized for HRMs are derived from pressure profiles from non-reactive experiments or simulations, which are void of the effects of chemical reaction. Therefore, the effects of preliminary chemical heat release cannot be accounted for in this approach. The modified MZM, which simulates chemical reaction throughout the entire temperature-varying main reaction chamber, accounts for the effects of chemical heat release directly throughout an entire simulation.

The modified MZM is shown to yield similar temperature, pressure, and chemical species profiles as CFD simulations for cases with neat and blended primary reference fuels, although more discrepancy between CFD and MZM data is seen for cases with longer ignition delays. Good agreement in mole fractions of fuel between MZM and 
CFD simulation is present in every case, although some discrepancy exists during combustion, due to differences in ignition delay time. Also, CFD predicts a second stage of $n$-heptane decomposition for the fuel-blend cases, while the MZM predicts only one. The amount of intermediate species mole fractions predicted by the MZM agree well with predictions from CFD, with the highest percent differences occurring for cases with higher compression ratios, which have the shortest ignition delay times. On average, the MZM over predicts radical species mole fractions by about $15 \%$ compared to CFD predictions, with the best agreement coming from the $n$-heptane cases which yield singlestage ignition, and the worst coming from the iso-octane cases which yield definite twostage ignition. The radical species concentrations predictions by the MZM were much closer than predictions by the HRM, which yielded percent differences with CFD ranging from $80-100 \%$. HRM simulations only model the core region, which has conditions that can be much different than the other areas of an RCM, preventing them from accurately predicting cylinder-averaged speciation data.

\subsection{Future Work}

One possibility for future work regarding the MZM could be to model the gas dynamic effects and mixing between the thermal boundary layer and core region. Another could be to address discrepancies in temperature and pressure that occur in simulations with long ignition delay times, perhaps specifically targeting the tapered gap and crevice models, which govern enthalpy loss from the main reaction chamber. A study which directly assesses the validity of the assumptions used to formulate the MZM (such as no convection within the main combustion chamber, quasi-steady flow through the tapered gap, only convective heat transfer in the gap, etc.) using CFD simulations 
would be interesting. Lastly, quenched RCM experiments for the conditions given in this study should be performed, and the speciation data obtained should be compared to results from this study in order to further evaluate the predictive accuracy of the MZM. It is likely that for the MZM to give comparable results to RCM experiments, an additional model to account for crevice blow-by would need to be added to the model. 


\section{BIBLIOGRAPHY}

Clarkson, J., Griffiths, J. F., MacNamara, J. P., \& Whitaker, B. J. (2001). Temperature fields during the development of combustion in a rapid compression machine. Combustion and Flame, 125(3), 1162-1175.

Costes, M., \& Naulin, C. (2013). Studies of reactions relevant to astrochemistry. Annual Reports Section "C" (Physical Chemistry), 109, 189.

Curran, H. J., Gaffuri, P., Pitz, W. J., \& Westbrook, C. K. (1998). A comprehensive modeling study of n-heptane oxidation. Combustion and Flame, 114(1-2), 149-177.

Dayma, G., Sarathy, S. M., Togbé, C., Yeung, C., Thomson, M. J., \& Dagaut, P. (2011). Experimental and kinetic modeling of methyl octanoate oxidation in an opposedflow diffusion flame and a jet-stirred reactor. Proceedings of the Combustion Institute, 33(1), 1037-1043.

Egolfopoulos, F. N., Hansen, N., Ju, Y., Kohse-H??inghaus, K., Law, C. K., \& Qi, F. (2014). Advances and challenges in laminar flame experiments and implications for combustion chemistry. Progress in Energy and Combustion Science, 43, 36-67.

Epping, K., Aceves, S., Bechtold, R., \& Dec, J. E. (2002). The Potential of HCCI Combustion for High Efficiency and Low Emissions. SAE Technical Paper, (724), 2002-01-1923.

Goldsborough, S. S., Banyon, C., \& Mittal, G. (2012). A computationally efficient, physics-based model for simulating heat loss during compression and the delay period in RCM experiments. Combustion and Flame, 159(12), 3476-3492.

Goldsborough, S. S., Mittal, G., \& Banyon, C. (2013). Methodology to account for multistage ignition phenomena during simulations of RCM experiments. Proceedings of the Combustion Institute, 34(1), 685-693.

Goldsborough, S. S., \& Potokar, C. J. (2007). The Influence of Crevice Flows and BlowBy on the Charge Motion and Temperature Profiles Within a Rapid Compression Expansion Machine Used for Chemical Kinetic (HCCI) Studies. SAE Technical Paper Series, (2007-01-0169), 776-790. http://doi.org/10.4271/2007-01-0169

Griffiths, J. F., MacNamara, J. P., Mohamed, C., Whitaker, B. J., Pan, J., \& Sheppard, C. G. (2001). Temperature fields during the development of autoignition in a rapid compression machine. Faraday Discussions, 119(119), 287-303; discussion 353370 .

Herbinet, O., Pitz, W. J., \& Westbrook, C. K. (2010). Detailed chemical kinetic mechanism for the oxidation of biodiesel fuels blend surrogate. Combustion and Flame, 157(5), 893-908. 
Heywood B. John. (1988). Internal Combustion Engine Fundamentals. McGraw-Hill Education.

Hughes, K. J., Turanyi, T., Clague, A. R., \& Pilling, M. J. (2001). Development and testing of a comprehensive chemical mechanism for the oxidation of methane. International Journal of Chemical Kinetics, 33(9), 513-538.

Kays, W. M., Crawford, M. E., \& Weigand, B. (2004). Convective Heat and Mass Transfer (4th ed.). McGraw-Hill Education.

Kong, S.-C., Sun, Y., \& Rietz, R. D. (2007). Modeling Diesel Spray Flame Liftoff, Sooting Tendency, and NO[sub x] Emissions Using Detailed Chemistry With Phenomenological Soot Model. Journal of Engineering for Gas Turbines and Power, 129(1), 245.

Kumar, K., Mittal, G., \& Sung, C. J. (2009). Autoignition of n-decane under elevated pressure and low-to-intermediate temperature conditions. Combustion and Flame, 156(6), 1278-1288.

Lee, D., \& Hochgreb, S. (1998). Rapid Compression Machines: Heat Transfer and Suppression of Corner Vortex. Combustion and Flame, 114(3-4), 531-545.

Liang, L., Reitz, R., Iyer, C., and Yi, J. (2007). Modeling Knock in Spark-Ignition Engines Using a G-equation Combustion Model Incorporating Detailed Chemical Kinetics. Sae, 2007-01-01(724), 776-0790.

Linse, D., Kleemann, A., \& Hasse, C. (2014). Probability density function approach coupled with detailed chemical kinetics for the prediction of knock in turbocharged direct injection spark ignition engines. Combustion and Flame, 161(4), 997-1014.

Mittal, G., Raju, M. P., \& Bhari, A. (2011). A numerical assessment of the novel concept of crevice containment in a rapid compression machine. Combustion and Flame, 158(12), 2420-2427.

Mittal, G., Raju, M. P., \& Sung, C. J. (2008). Computational fluid dynamics modeling of hydrogen ignition in a rapid compression machine. Combustion and Flame, 155(3), 417-428.

Mittal, G., Raju, M. P., \& Sung, C. J. (2010). CFD modeling of two-stage ignition in a rapid compression machine: Assessment of zero-dimensional approach. Combustion and Flame, 157(7), 1316-1324.

Mittal, G., Raju, M. P., \& Sung, C.-J. (2012). Vortex formation in a rapid compression machine: Influence of physical and operating parameters. Fuel, 94, 409-417.

Mittal, G., \& Sung, C.-J. (2006). Aerodynamics inside a rapid compression machine. Combustion and Flame, 145(1-2), 160-180. 
Mittal, G., \& Sung, C.-J. (2007). A Rapid Compression Machine for Chemical Kinetics Studies At Elevated Pressures and Temperatures. Combustion Science and Technology, 179(3), 497-530.

Mueller, M. A., Yetter, R. A., \& Dryer, F. L. (1999). Flow reactor studies and kinetic modeling of the $\mathrm{H} 2 / \mathrm{O} 2 / \mathrm{NOx}$ and $\mathrm{CO} / \mathrm{H} 2 \mathrm{O} / \mathrm{O} 2 / \mathrm{NOx}$ reactions. International Journal of Chemical Kinetics, 31(10), 705-724.

Neuman, J. (2015). Development of a Rapid Compression Controlled-Expansion Machine for Chemical Ignition Studies. Marquette University.

Petersen, E. L., Davidson, D. F., \& Hanson, R. K. (1999). Kinetics modeling of shockinduced ignition in low-dilution $\mathrm{CH} 4 / \mathrm{O} 2$ mixtures at high pressures and intermediate temperatures. Combustion and Flame, 117(1-2), 272-290.

Ranzi, E., Frassoldati, A., Grana, R., Cuoci, A., Faravelli, T., Kelley, A. P., \& Law, C. K. (2012). Hierarchical and comparative kinetic modeling of laminar flame speeds of hydrocarbon and oxygenated fuels. Progress in Energy and Combustion Science, $38(4), 468-501$.

Reactor Networks. (2012). Retrieved March 1, 2016, from http://www.cantera.org/docs/sphinx/html/reactors.html

Ribaucour, M., Minetti, R., Sochet, L. R., Curran, H. J., Pitz, W. J., \& Westbrook, C. K. (2000). Ignition of isomers of pentane: An experimental and kinetic modeling study. Proceedings of the Combustion Institute, 28(2), 1671-1678.

Richards, K. J., Senecal, P. K., A., \& Pomraning, E. (2014). CONVERGE(V2.2.0). Madison, WI: Convergent Science, Inc.

Saxena, S., \& Bedoya, I. D. (2013). Fundamental phenomena affecting low temperature combustion and HCCI engines, high load limits and strategies for extending these limits. Progress in Energy and Combustion Science, 39(5), 457-488.

Simmie, J. M. (2003). Detailed chemical kinetic models for the combustion of hydrocarbon fuels. Progress in Energy and Combustion Science, 29(6), 599-634.

Som, S., Ramirez, A. I., Longman, D. E., \& Aggarwal, S. K. (2011). Effect of nozzle orifice geometry on spray, combustion, and emission characteristics under diesel engine conditions. Fuel, 90(3), 1267-1276.

Sung, C.-J., \& Curran, H. J. (2014). Using rapid compression machines for chemical kinetics studies. Progress in Energy and Combustion Science, 44, 1-18.

Tanaka, S., Ayala, F., \& Keck, J. C. (2003). A reduced chemical kinetic model for HCCI combustion of primary reference fuels in a rapid compression machine. Combustion and Flame, 133(4), 467-481. 
Tiwary, P., Limongelli, V., Salvalaglio, M., \& Parrinello, M. (2015). Kinetics of proteinligand unbinding: Predicting pathways, rates, and rate-limiting steps. Proceedings of the National Academy of Sciences, 112(5), 201424461.

Tsurushima, T. (2009). A new skeletal PRF kinetic model for HCCI combustion. Proceedings of the Combustion Institute, 32 II(February), 2835-2841. http://doi.org/10.1016/j.proci.2008.06.018

Turns, S. R. (2012). An Introduction to Combustion: Concepts and Applications (3rd ed.). McGraw-Hill Education.

Vanhove, G., Yu, Y., Boumehdi, M., Frottier, O., Herbinet, O., Glaude, P.-A., \& BattinLeclerc, F. (2015). Experimental study of tetrahydrofuran oxidation and ignition in low-temperature conditions. Energy \& Fuels.

Villafruela, J. M., Castro, F., \& Parra, M. T. (2008). Experimental study of parallel and inclined turbulent wall jets. Experimental Thermal and Fluid Science, 33(1), 132139.

Wagnon, S. W., Barraza-Botet, C. L., \& Wooldridge, M. S. (2015). Effects of bond location on the ignition and reaction pathways of trans-hexene isomers. Journal of Physical Chemistry A, 119(28), 7695-7703. http://doi.org/10.1021/acs.jpca.5b01029

Westbrook, C. K., Pitz, W. J., Boercker, J. E., Curran, H. J., Griffiths, J. F., Mohamed, C., \& Ribaucour, M. (2002). Detailed chemical kinetic reaction mechanisms for autoignition of isomers of heptane under rapid compression. Proceedings of the Combustion Institute, 29(1), 1311-1318.

Würmel, J., \& Simmie, J. (2005). CFD studies of a twin-piston rapid compression machine. Combustion and Flame, 141(4), 417-430.

Yasunaga, K., Mikajiri, T., Sarathy, S. M., Koike, T., Gillespie, F., Nagy, T., ... Curran, H. J. (2012). A shock tube and chemical kinetic modeling study of the pyrolysis and oxidation of butanols. Combustion and Flame, 159(6), 2009-2027. 